\title{
Integrable and Chaotic Dynamics of Spins Coupled to an Optical Cavity
}

\author{
Gregory Bentsen, ${ }^{1,4, *}$ Ionut-Dragos Potirniche ${ }^{2,{ }^{*}}$ Vir B. Bulchandani, ${ }^{2}$ Thomas Scaffidi, ${ }^{2,5}$ Xiangyu Cao, ${ }^{2}$ \\ Xiao-Liang Qi ${ }^{1}$ Monika Schleier-Smith, ${ }^{1,4}$ and Ehud Altman ${ }^{2,3}$ \\ ${ }^{1}$ Department of Physics, Stanford University, Stanford, California 94305, USA \\ ${ }^{2}$ Department of Physics, University of California, Berkeley, Berkeley, California 94720, USA \\ ${ }^{3}$ Materials Science Division, Lawrence Berkeley National Laboratory, Berkeley, California 94720, USA \\ ${ }^{4}$ SLAC National Accelerator Laboratory, Menlo Park, California 94025, USA \\ ${ }^{5}$ Department of Physics, University of Toronto, Toronto, Ontario, M5S 1A7, Canada
}

(Received 6 May 2019; revised manuscript received 21 July 2019; published 15 October 2019)

\begin{abstract}
We show that a class of random all-to-all spin models, realizable in systems of atoms coupled to an optical cavity, gives rise to a rich dynamical phase diagram due to the pairwise separable nature of the couplings. By controlling the experimental parameters, one can tune between integrable and chaotic dynamics on the one hand and between classical and quantum regimes on the other hand. For two special values of a spin-anisotropy parameter, the model exhibits rational Gaudin-type integrability, and it is characterized by an extensive set of spin-bilinear integrals of motion, independent of the spin size. More generically, we find a novel integrable structure with conserved charges that are not purely bilinear. Instead, they develop "dressing tails" of higher-body terms, reminiscent of the dressed local integrals of motion found in many-body localized phases. Surprisingly, this new type of integrable dynamics found in finitesize spin- $1 / 2$ systems disappears in the large- $S$ limit, giving way to classical chaos. We identify parameter regimes for characterizing these different dynamical behaviors in realistic experiments, in view of the limitations set by cavity dissipation.
\end{abstract}

DOI: 10.1103/PhysRevX.9.041011

\section{INTRODUCTION}

The dynamics leading to the eventual thermalization of closed quantum systems has become a topic of intense interest over the past few years. Significant progress has been made in describing the scrambling of information through quantum chaos, which allows effectively irreversible dynamics to emerge from unitary quantum time evolution. Notably, Maldacena et al., inspired by the chaotic properties of black holes, established that quantum mechanics places an upper bound on the Lyapunov exponent that characterizes the growth of chaos [1]. In a related development, Kitaev constructed a class of quantum many-body models whose dynamics saturates this bound on chaos [2,3] and can be related to black holes through the AdS/CFT correspondence [4-6]. The fact that these models admit controlled solutions, despite being chaotic, has further conferred on them a paradigmatic status within the field of quantum dynamics.

\footnotetext{
*These authors contributed equally to the paper.
}

Published by the American Physical Society under the terms of the Creative Commons Attribution 4.0 International license. Further distribution of this work must maintain attribution to the author(s) and the published article's title, journal citation, and DOI.
Subject Areas: Atomic and Molecular Physics, Statistical Physics

Finding accessible systems that realize such models is therefore highly desirable but also a priori very challenging: A common feature shared by all of these maximally chaotic, holographic models is that they lack spatial locality since they couple together an extensive number of degrees of freedom (d.o.f.). For instance, the Sachdev-Ye (SY) model [7] was originally proposed as a quantum spin model with random all-to-all couplings:

$$
H=\frac{1}{\sqrt{N M}} \sum_{i, j=1}^{N} U_{i j} \mathbf{S}_{i} \cdot \mathbf{S}_{j},
$$

where $\mathbf{S}_{i}$ are $\mathrm{SU}(M)$ spin operators. A fermionic variant, the Sachdev-Ye-Kitaev (SYK) model, was subsequently introduced by Kitaev [2].

While infinite-range spin interactions do not occur in magnetic materials, they can be realized rather naturally in cold atomic ensembles coupled to an optical cavity mode [8-22]. In this setup, the delocalized cavity mode mediates infinite-range interactions between the internal states of atoms through the local coupling at each site, regardless of the distance between atoms [23-31]. However, there is a crucial difference, already pointed out in Ref. [32], between the interactions in the SY model and the ones mediated by the cavity. The second-order process that couples the atomic d.o.f. via the cavity mode gives a separable (rank-1) 


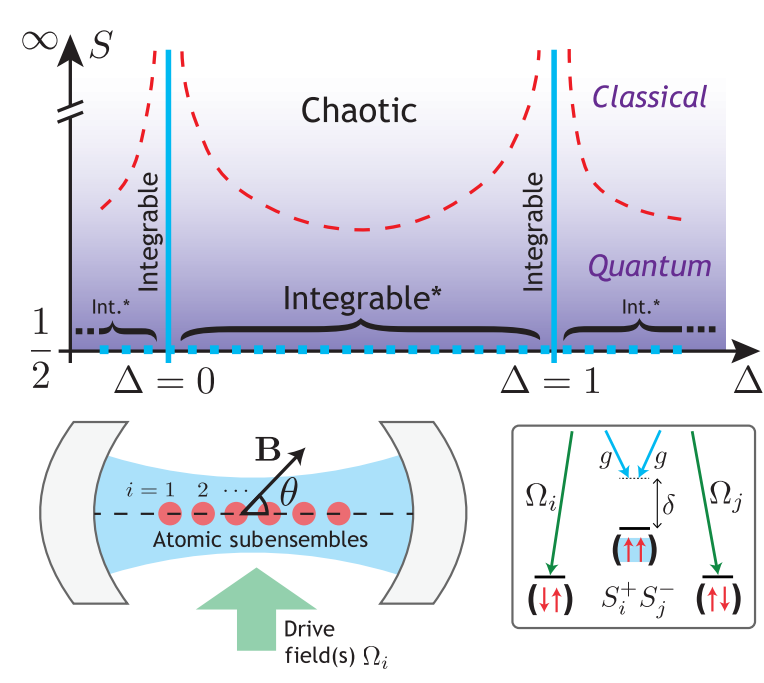

FIG. 1. (Top panel) The dynamical phase diagram of the model (1.2) in the plane of spin size $S$ and anisotropy $\Delta$. The main features are as follows: the integrable lines at $\Delta=0$ and $\Delta=1$ (solid blue lines), the novel integrable line at $S=1 / 2$ (dotted blue line and marked by an asterisk), and the onset of chaos at large $S$, indicated schematically by the dashed red curves. (Bottom-left panel) Schematic of the atomic subensembles (red) trapped inside a single-mode optical cavity (blue). A drive field (green) at a detuning $\delta$ from the cavity resonance generates effective spin-spin interactions between the atoms (bottom-right panel). The tunable angle $\theta$ between the spin quantization axis (along the applied magnetic field $\mathbf{B}$ ) and the cavity's longitudinal axis leads to an anisotropy $\Delta=2 \cot ^{2} \theta$. By changing the local atomic density in a region of constant coupling to the cavity mode, the effective spin size $S$ can also be varied, allowing for the systematic exploration of the full phase diagram.

matrix $U_{i j}=J_{i} J_{j}$, rather than the full-rank matrix assumed in the different variants of the SY model. Although nonseparable interactions are, in principle, accessible in multimode cavities [14,15,20,26,32-34], separable all-to-all couplings are realized in numerous existing experiments $[10,13,16-19,21,22]$ and arise generically for interactions mediated by a single bosonic mode.

Moreover, this ostensible limitation of the optical cavity scheme turns out to be a boon: The separability of the coupling matrix $U_{i j}$ is responsible for an even richer dynamical phase diagram (see Fig. 1), which includes regions of chaos, Gaudin-type integrability characterized by spin-bilinear conserved quantities, and of a novel form of integrability-labeled integrable* in Fig. 1-with quasibilinear integrals of motion.

The class of models we consider in this paper is described by the following quantum spin Hamiltonian:

$$
H=\frac{1}{S \sqrt{N}} \sum_{i, j=1}^{N} J_{i} J_{j}\left(S_{i}^{x} S_{j}^{x}+S_{i}^{y} S_{j}^{y}+\Delta S_{i}^{z} S_{j}^{z}\right),
$$

where $\mathbf{S}_{i}$ are $\mathrm{SU}(2)$ spin-S operators encoded in the magnetic sublevels of individual atoms or atomic subensembles located at sites $i=1, \ldots, N$. The site-dependent coefficients $J_{i}$ are determined by the local coupling of the atoms at site $i$ to the spatially varying cavity mode or by the local Rabi frequency $\Omega_{i}$ of an inhomogeneous drive field. The nonuniformity of the couplings $J_{i}$ is a crucial element of the models under consideration. For perfectly uniform couplings $\left(J_{i}=J\right)$, the model is integrable and exactly solvable in terms of the macroscopic spin $\mathcal{F} \propto \sum_{i} J_{i} \mathbf{S}_{i}$. The $S^{x} S^{x}$ and $S^{y} S^{y}$ terms in Eq. (1.2) describe spin-exchange interactions between pairs of atoms, mediated by virtual cavity photons, while the $S^{z} S^{z}$ terms describe statedependent ac Stark shifts. The normalization of $H$, which is not important for the dynamical properties, ensures that the high-temperature specific heat and free energy have a proper thermodynamic limit [35].

The dynamical phases generated by this nonlocal spin model, shown in Fig. 1, are accessible via two experimentally tunable parameters. The spin-anisotropy parameter $\Delta$, controlling the relative strength of the spin-exchange and $S^{z} S^{z}$ interactions, can be tuned by changing the angle of an applied magnetic field $\mathbf{B}$ (see Fig. 1). In addition, it is possible to control the strength of quantum effects by changing the spin size $S$ on each site. While the choice of internal atomic states provides some flexibility in varying $S$, a larger range of spin sizes can be achieved by varying the number of atoms trapped at each site and letting $S_{i}^{\alpha}$ represent the collective spin of the subensemble at site $i$. This experimental control of the spin size $S$ enables the tuning of quantum effects from semiclassical dynamics at large $S$ all the way down to a spin- $1 / 2$ system that is dominated by quantum fluctuations. In combination with the possibility of varying the anisotropy $\Delta$, this tunability allows for a thorough exploration of the dynamical phase diagram.

The paper and the presentation of the various regimes shown in Fig. 1 are organized as follows. We provide a brief overview of these dynamical phases in Sec. II, and we emphasize the novel features, which constitute our main results. In Sec. III, we describe in detail the proposed experimental scheme to realize and control the couplings of the Hamiltonian (1.2). We also describe ways of inducing perturbations that go beyond separable interactions. In Sec. IV, we begin the derivation of the main results. We analytically construct the integrals of motion that demonstrate the integrability of the dynamics at the special points $\Delta=0$ and $\Delta=1$. In Sec. V, we present a computational method for finding integrals of motion using numerical or experimental data. In Sec. VI, we deploy this technique and provide numerical evidence for the existence of a novel quantum integrable regime away from the special points $\Delta=0,1$. Specifically, we present an exact diagonalization study of the spin-1/2 model, showing that the integrable structure persists for anisotropy values $\Delta \neq 0,1$ away from the integrable points, with quasibilinear integrals of motion. In Sec. VII, we simulate the classical model $(S \rightarrow \infty)$ and show that it becomes chaotic, albeit in the presence of 
slowly decaying modes, away from the special points. In Sec. VIII, we discuss experimental limitations and assess the extent to which the various features of the model are accessible in the presence of dissipation. Finally, in Sec. IX, we comment on the implications of these results before concluding.

\section{OVERVIEW OF THE PHASE DIAGRAM}

The best-understood part of the dynamical phase diagram in Fig. 1 is the line at $\Delta=1$, for all spin sizes $S$, on which the Hamiltonian from Eq. (1.2) is equivalent to a rational Gaudin model [36]. This model is quantum integrable in the mathematical sense of possessing an underlying quantum group structure [37]. In the context of Gaudin-type models, quantum integrability is characterized by the existence of an extensive family of commuting, bilinear conserved quantities, and there exist analytical expressions for each one. Even though there is no notion of spatial locality, the conserved quantities are "2-local" in the complexity theory sense [38,39]. By interchanging commutators with Poisson brackets, it follows that the integrable structure persists in the classical limit.

We find that the model is integrable at $\Delta=0$ as well. We obtain analytical expressions for an extensive family of conserved quantities that are also bilinear in spin. As in the case of $\Delta=1$, this integrability holds for any value of $S$, including the classical limit $S \rightarrow \infty$. The integrability of the model at $\Delta=0$ is connected to the existence of a nonstandard class of Gaudin models [40-47].

However, the most surprising part of the phase diagram occurs away from these integrable points, i.e., in the regions $\{\Delta<0\},\{0<\Delta<1\}$, and $\{\Delta>1\}$. There, we find a novel integrable structure that is markedly different from the type of integrability found at the two integrable points, $\Delta=0$ and $\Delta=1$. First, unlike the latter, integrability for $\Delta \neq 0,1$ appears to depend crucially on the spin size $S$. We show strong evidence that the model is integrable for a spin- $1 / 2$ system, while it is chaotic with a finite Lyapunov exponent $\lambda$ in the classical limit $(S \rightarrow \infty)$. Nevertheless, in this latter limit, we also find that there exist modes that relax only on timescales much larger than $\lambda^{-1}$. We conjecture that this is a consequence of the "quasi-integrable" nature [48] of the classical model. The putative transition from quantum integrable to (semiclassical) chaotic dynamics, schematically shown in Fig. 1, can be probed experimentally.

Second, the integrals of motion (IOM) of the $S=1 / 2$ model at $\Delta \neq 0,1$ are not bilinear (or 2-local) but may instead be termed "quasibilinear." We present compelling numerical evidence that each IOM has appreciable support in the space of bilinear spin operators that does not depend on the system size $N$. The fact that the integrals of motion persist while developing tails of multispin terms on top of the dominant two-spin contribution is reminiscent of the quasilocal integrals of motion that characterize many-body localized (MBL) phases [49-53].

\section{PROPOSED EXPERIMENTAL SCHEME}

As advertised, the full phase diagram shown in the top panel of Fig. 1 can be accessed in experiments with atomic ensembles in single-mode optical cavities. In such experiments, each spin is encoded in internal states of an individual atom. The cavity generically couples to a weighted collective spin

$$
\mathcal{F} \equiv \sum_{i} \xi_{i} \mathbf{S}_{i}
$$

where each weight $\xi_{i}$ is set by the amplitudes of the cavity mode and drive field at the position of the $i$ th atom. Experiments to date have realized either Ising interactions [10,13,17,18,22] $H \propto \mathcal{F}_{z}^{2}$ or spin-exchange interactions $[16,21] H \propto \mathcal{F}_{+} \mathcal{F}_{-}$, in the latter case directly imaging the spatial dependence of the weights $\xi_{i}$ and the resulting spin dynamics [21]. We now show how to extend the approach of Ref. [21] to realize generic $X X Z$ models of the form

$$
H \propto \mathcal{F}_{x} \mathcal{F}_{x}+\mathcal{F}_{y} \mathcal{F}_{y}+\Delta \mathcal{F}_{z} \mathcal{F}_{z},
$$

where the anisotropy $\Delta$ is tuned by the angle of a magnetic field. An alternative approach to engineering Heisenberg models has been proposed in Ref. [31].

The experimental setup proposed here is shown in the bottom-left panel of Fig. 1. We consider spins encoded in Zeeman states of atoms whose positions in the cavity are fixed by a deep optical lattice. A magnetic field $\mathbf{B}=B \hat{z}$, which defines the quantization axis for the spins, is oriented at an angle $\theta$ to the longitudinal axis $\hat{c}$ of the optical cavity. Driving the atoms with a control field, incident either through the cavity or from the side, allows pairs of atoms to interact by scattering photons via the cavity. The interaction strengths are governed by the spatially dependent Rabi frequency $\Omega_{i}$ of the control field and vacuum Rabi frequency $2 g_{i}$ of the cavity, where $i$ denotes the value for the $i$ th atom.

For large detuning between the atomic and cavity resonances, the atom-cavity interaction takes the form of a Faraday effect in which each atom couples to the Stokes vector $\mathbf{I}_{i}$, representing the local polarization and intensity of light. This effect is described by a Hamiltonian

$$
H_{I}=2 \chi \sum_{i}\left(\mathbf{I}_{i} \cdot \hat{c}\right)\left(\mathbf{S}_{i} \cdot \hat{c}\right)
$$

where $\chi$ is the vector ac Stark shift of a maximally coupled atom and the component of the Stokes vector along the cavity is $\mathbf{I}_{i} \cdot \hat{c}=\left(A_{+, i}^{\dagger} A_{+, i}-A_{-, i}^{\dagger} A_{-, i}\right) / 2$. The field operators

$$
A_{ \pm, i}=\left(\frac{\Omega_{i} e^{-i \delta t}}{2 \sqrt{2}}+g_{i} a_{ \pm}\right) / g
$$

include the quantum field $a_{ \pm}$of the cavity for $\sigma_{ \pm}-$ polarized modes, weighted by the local amplitude $g_{i}$ 
of the cavity mode and displaced by a classical drive field with local Rabi frequency $\Omega_{i}$. The normalization is set by the vacuum Rabi frequency $2 g$ of a maximally coupled atom. We assume that the drive field has horizontal polarization $\hat{x}=\hat{z} \times \hat{c}$ and is detuned by $\delta$ from the cavity resonance.

In the limit where the drive field is weak and far detuned, we can obtain an effective Hamiltonian for the spin dynamics by adiabatically eliminating the photon modes. To this end, we first expand $H_{I}$ to lowest order in the operators $a_{ \pm}$to obtain

$$
H_{I} \approx \frac{i}{2} \chi\left(\xi_{i}^{*} v e^{i \delta t}-\xi_{i} v^{\dagger} e^{-i \delta t}\right)\left(\mathbf{S}_{i} \cdot \hat{c}\right)
$$

where $v=\left(a_{+}-a_{-}\right) / \sqrt{2}$ represents the vertically polarized cavity mode, and we have introduced the weights

$$
\xi_{i}=\frac{\Omega_{i} g_{i}^{*}}{g^{2}}
$$

These weights determine the collective spin $\mathcal{F}$ defined in Eq. (3.1), which couples to the cavity mode. Then, for $\left\langle v^{\dagger} v\right\rangle \ll 1$ and for large detuning $\delta \gg \kappa, \omega_{Z}$ compared to the cavity linewidth $\kappa$ and Zeeman splitting $\omega_{Z}$, we find that the effective spin Hamiltonian is [35]

$H=\frac{\chi^{2}}{4 \delta}\left[\cos ^{2} \theta \mathcal{F}_{z} \mathcal{F}_{z}+\frac{1}{2} \sin ^{2} \theta\left(\mathcal{F}_{x} \mathcal{F}_{x}+\mathcal{F}_{y} \mathcal{F}_{y}\right)\right]$.

We see that Eq. (3.7) matches the Hamiltonian (1.2) with couplings $J_{i}=\chi \xi_{i} S^{1 / 2} N^{1 / 4} \sin \theta / 2 \sqrt{2 \delta}$ and anisotropy $\Delta=2 \cot ^{2} \theta$. Note that arbitrary control over the set of weights $\xi_{i}$ can be obtained by designing the spatial dependence of the control field.

In addition to the coherent dynamics generated by $H$ from Eq. (3.7), the cavity-mediated interactions are subject to dissipation due to photon loss from the cavity mirrors and atomic free-space scattering. Formally, these processes can be described by a family of Lindblad operators acting within a quantum master equation [35]. The key parameter governing the interaction-to-decay ratio is the single-atom cooperativity $\eta=4 g^{2} / \kappa \Gamma$, where $\Gamma$ is the atomic excitedstate linewidth. Moreover, we find that the interactionto-decay ratio is collectively enhanced, scaling as $S \sqrt{N \eta}$ for a system of $N$ subensembles consisting of $S$ atoms each.

After discussing the various properties and measurable signatures of chaotic and integrable dynamics in Eq. (1.2), we return to quantifying the effects of dissipation in Sec. VIII. In particular, we estimate the atom number and cooperativity $\eta$ requisite for observing these signatures in the experimental setup.

\section{INTEGRABILITY AT $\Delta=0$ AND $\Delta=1$}

In this section, we demonstrate the quantum integrability of the Hamiltonian (1.2) along the two lines at $\Delta=0$ and $\Delta=1$ in the dynamical phase diagram (Fig. 1). To place our discussion in context for the nonspecialist reader, we begin by recalling some key features of integrable many-body systems. Broadly speaking [54], such systems are characterized by an extensive number of local conservation laws that give rise to exotic transport and thermalization properties. Important examples of quantum integrable systems include the Lieb-Liniger Bose gas and the spin-1/2 Heisenberg chain.

To illustrate the main ideas, consider a one-dimensional, local, quantum Hamiltonian $H=\sum_{n=1}^{N} h_{n}$, on $N$ lattice sites. For this type of model, integrability means the existence of $N-1$ independent, local charges,

$$
Q^{(n)}=\sum_{i=1}^{N} q_{i}^{(n)}, \quad n=2, \ldots, N,
$$

that commute with each other and with the Hamiltonian, namely,

$$
\left[Q^{(m)}, Q^{(n)}\right]=0, \quad\left[Q^{(m)}, H\right]=0 .
$$

The existence of extensively many local conservation laws can be regarded as a strong constraint on the dynamics of such systems and leads to unusual physical effects such as nondissipative heat transport [56] and equilibration to nonthermal steady states $[57,58]$.

In contrast with more standard integrable systems, the Gaudin-type models that arise in the present work are somewhat unusual since they exhibit nonlocal couplings and are therefore essentially zero dimensional. To construct these models, one starts from a set of $N$ operators,

$$
G^{(i)}=\sum_{j=1}^{N} \sum_{\alpha=1}^{3} w_{i j}^{\alpha} S_{i}^{\alpha} S_{j}^{\alpha}, \quad i=1,2, \ldots, N,
$$

that are linear combinations of spin bilinears, with real coefficients $w_{i j}^{\alpha} \in \mathbb{R}$, and that satisfy the defining commutation relations:

$$
\left[G^{(i)}, G^{(j)}\right]=0 .
$$

The physical Hamiltonian and the independent conserved charges are then given by linear combinations of the $G^{(i)}$, of the form

$$
H=\sum_{i=1}^{N} a_{i}^{(0)} G^{(i)}, \quad Q^{(n)}=\sum_{i=1}^{N} a_{i}^{(n)} G^{(i)}, \quad n=2, \ldots, N,
$$


where the coefficients $a_{i}^{(n)} \in \mathbb{R}$ are elements of a nonsingular $N \times N$ matrix. Note that by the commutation relations (4.4), the Hamiltonian $H$ and its associated charges $Q^{(n)}$ automatically satisfy the commutation relations (4.2) required for integrability. Although these operators are not local, they are sums of spin bilinears and can therefore be regarded as 2-local in the complexity theory sense.

We now show that the Hamiltonian Eq. (1.2) defines a Gaudin-type integrable model for $\Delta=0$ and $\Delta=1$ and all values of spin $S$. Specifically, we demonstrate that along these lines in the dynamical phase diagram Fig. 1, there exist $N-1$ independent, conserved, and mutually commuting spin bilinears. The Hamiltonian at $\Delta=1$ is related to the rational Gaudin model [36], which is well known to be quantum integrable in the mathematically rigorous sense of possessing an underlying quantum group structure [37]. Meanwhile, the Hamiltonian at $\Delta=0$ lies in a less wellknown class of "nonskew" Gaudin models, which arise from Gaudin's equations upon relaxing the constraint of antisymmetry under the interchange of site indices [40-47].

It will be helpful to review the problem first studied by Gaudin [36]: Under what circumstances do a set of spin bilinears, as in Eq. (4.3), define a mutually commuting set, with $\left[G^{(i)}, G^{(j)}\right]=0$ ? If the couplings $w_{i j}^{\alpha} \in \mathbb{R}$ are taken to be antisymmetric under interchange of indices, with $w_{i j}^{\alpha}+w_{j i}^{\alpha}=0$, then the $G^{(i)}$ mutually commute if and only if the Gaudin equations

$$
w_{i j}^{\alpha} w_{j k}^{\gamma}+w_{j i}^{\beta} w_{i k}^{\gamma}-w_{i k}^{\alpha} w_{j k}^{\beta}=0
$$

hold for all pairwise distinct $\{i, j, k\}$ and $\{\alpha, \beta, \gamma\}$. The isotropic solution $w_{i j}^{\alpha}=J_{i} J_{j} /\left(J_{i}-J_{j}\right)$ defines the rational Gaudin Hamiltonians

$$
G^{(i)}(\vec{J})=\sum_{j \neq i} \frac{J_{i} J_{j}}{J_{i}-J_{j}} \mathbf{S}_{i} \cdot \mathbf{S}_{j}
$$

The all-to-all spin model from Eq. (1.2) at $\Delta=1$ is simply a linear combination of rational Gaudin Hamiltonians and Casimirs, to wit

$H=\sum_{i, j=1}^{N} J_{i} J_{j} \mathbf{S}_{i} \cdot \mathbf{S}_{j}=\sum_{i=1}^{N} 2 J_{i} G^{(i)}(\vec{J})+J_{i}^{2} \mathbf{S}_{i} \cdot \mathbf{S}_{i}$.

By rotational symmetry, $H$ conserves the total spin $\mathbf{S}_{\mathrm{tot}}=\sum_{i} \mathbf{S}_{i}$, and the linear span of the $G^{(i)}(\vec{J})$ includes the squared spin $\mathbf{S}_{\mathrm{tot}} \cdot \mathbf{S}_{\mathrm{tot}}=\sum_{i, j} \mathbf{S}_{i} \cdot \mathbf{S}_{j}$. The mathematical structure of traditional Gaudin models has been studied in depth $[37,59]$.

Let us now consider relaxing the constraint of antisymmetric couplings. Then, Gaudin's equations (4.6) must be augmented by two equations constraining "on-site" couplings, which read

$$
\begin{aligned}
& \left(w_{i j}^{\beta} w_{j i}^{\gamma}-w_{j i}^{\beta} w_{i j}^{\gamma}\right)+2 w_{j i}^{\alpha}\left(w_{i i}^{\gamma}-w_{i i}^{\beta}\right)=0, \\
& \left(w_{i j}^{\alpha} w_{j i}^{\gamma}-w_{j i}^{\alpha} w_{i j}^{\gamma}\right)+2 w_{j i}^{\beta}\left(w_{i i}^{\gamma}-w_{i i}^{\alpha}\right)=0 .
\end{aligned}
$$

The model from Eq. (1.2) at $\Delta=0$ arises from a "nonskew $X X Z$ " solution $w_{i j}^{1}=w_{i j}^{2}=J_{i} J_{j} /\left(J_{i}^{2}-J_{j}^{2}\right), w_{i j}^{3}=J_{j}^{2} /\left(J_{i}^{2}-J_{j}^{2}\right)$ to the usual Gaudin equation (4.6), augmented by on-site terms $w_{i i}^{1}=w_{i i}^{2}=1 / 2, w_{i i}^{3}=0$, which solve Eq. (4.9). The corresponding Gaudin Hamiltonians read

$$
\begin{aligned}
\tilde{G}^{(i)}(\vec{J})= & \sum_{j \neq i} \frac{J_{i} J_{j}}{J_{i}^{2}-J_{j}^{2}}\left(S_{i}^{x} S_{j}^{x}+S_{i}^{y} S_{j}^{y}\right)+\frac{J_{j}^{2}}{J_{i}^{2}-J_{j}^{2}} S_{i}^{z} S_{j}^{z} \\
& +\frac{1}{2}\left(S_{i}^{x} S_{i}^{x}+S_{i}^{y} S_{i}^{y}\right) .
\end{aligned}
$$

By the Gaudin equations (4.6) and (4.9), these mutually commute, and the Hamiltonian (1.2) at $\Delta=0$ can be expressed as

$$
H=\sum_{i, j=1}^{N} J_{i} J_{j}\left(S_{i}^{x} S_{j}^{x}+S_{i}^{y} S_{j}^{y}\right)=\sum_{i=1}^{N} 2 J_{i}^{2} \tilde{G}^{(i)}(\vec{J}) .
$$

At spin $1 / 2$, this result coincides with the Hamiltonian obtained in Ref. [46] or the "Wishart-SYK" model [60], and consequently, it can be derived as a special case of the integrable spin- $1 / 2$ Hamiltonians considered in the recent work Ref. [47]. The integrability of Eq. (4.11) for arbitrary spin $S$ was first discussed in Refs. [43-45] (see also the references therein). We conclude that there is an integrable line in the phase diagram of the model (1.2) at $\Delta=0$. By rotational symmetry about the $z$ axis, this Hamiltonian conserves $S_{\mathrm{tot}}^{z}=\sum_{i} S_{i}^{z}$, and $\left(S_{\mathrm{tot}}^{z}\right)^{2}$ lies in the linear span of the $\tilde{G}^{(i)}(\vec{J})$. Finally, we note that upon replacing commutators with Poisson brackets in the derivation of the Gaudin equations, the integrable structure identified for $\Delta=0$ and $\Delta=1$ remains unaltered in the classical limit $(S \rightarrow \infty)$ of the Hamiltonian.

\section{EXTRACTING INTEGRALS OF MOTION FROM NUMERICAL OR EXPERIMENTAL DATA}

Having characterized the integrable structure for $\Delta=0$ and $\Delta=1$, it is natural to ask whether the integrability* of Eq. (1.2) extends to other, more generic values of the anisotropy: Can we find similar extensive sets of commuting bilinear conserved charges for $\Delta \neq 0,1$ ? To tackle this question in the absence of analytical tools, such as those used in the previous section, we develop a numerical method that enables the systematic search for bilinear (2-local) IOM. We emphasize that this novel technique can be applied to either numerical or experimental data. 
Let us first define a set of 2-local operators $\left\{\hat{O}_{a}\right\}$ :

$$
\hat{O}_{a} \equiv \frac{3}{S(S+1)} \hat{S}_{i}^{\alpha} \hat{S}_{j}^{\alpha},
$$

where $i>j$ and the index $a$ is a shorthand notation for $(i, j, \alpha)$. We note that this family of $3 N(N-1) / 2$ operators defines an orthonormal set with respect to the infinitetemperature inner product:

$$
\frac{1}{\mathcal{D}} \operatorname{Tr}\left[\hat{O}_{a}^{\dagger} \hat{O}_{b}\right]=\delta_{a b},
$$

where $\mathcal{D} \equiv \operatorname{Tr}[\mathbf{1}]=(2 S+1)^{N}$ is the dimension of the Hilbert space.

Now, suppose that we can measure, experimentally or numerically, the time evolution of the expectation value $\left\langle\hat{O}_{a}(t)\right\rangle \equiv\left\langle\Phi\left|\hat{O}_{a}(t)\right| \Phi\right\rangle$, where $|\Phi\rangle$ is a random initial state (i.e., far from any energy eigenstate). A bilinear integral of motion $\hat{I}$ is a special linear combination of the $\hat{O}_{a}$ that remains constant in time, to wit

$$
\langle\hat{I}\rangle=\langle\hat{I}(t)\rangle \equiv \sum_{a} u_{a}\left\langle\hat{O}_{a}(t)\right\rangle=\sum_{a} u_{a} \overline{\left\langle\hat{O}_{a}\right\rangle} .
$$

Here and below, the overline denotes a time average, such as $\overline{\left\langle\hat{O}_{a}\right\rangle} \equiv \int_{0}^{T}[(d t) / T]\left\langle\hat{O}_{a}(t)\right\rangle$ over a time interval $[0, T]$. It is useful to recast the above equation in terms of the following time series matrix:

$$
M_{a, t} \equiv \sqrt{\frac{1}{T}}\left(\left\langle\hat{O}_{a}(t)\right\rangle-\overline{\left\langle\hat{O}_{a}\right\rangle}\right) .
$$

Note that $M_{a, t}$ is a rectangular matrix with $3 N(N-1) / 2$ rows and a continuum of columns indexed by $t \in[0, T]$, where $T J^{2} \gg 1$. In practice, the time axis is discretized such that the number of columns in $M$ is much larger than the number of rows. We immediately see that, by Eq. (5.3), a 2-local IOM corresponds to a left zero mode of $M$, i.e., $\sum_{a} u_{a} M_{a, t}=0$ for any $t$.

Thus, to find bilinear IOMs, we want to search for zero modes of $M$. More generally, we can consider the singular value decomposition (SVD) of $M$ or, equivalently, the spectrum of the real Hermitian matrix,

$$
\begin{aligned}
L_{a, b} & \equiv M M^{\dagger}=\int_{0}^{T} \frac{d t}{T} M_{a, t} M_{b, t} \\
& =\sum_{l=1}^{3 N(N-1) / 2} \sigma_{l}^{2} u_{a, l} u_{b, l} .
\end{aligned}
$$

In the second line, $\sigma_{l} \geq 0$ are the corresponding singular values of $M$, and $\sigma_{l}^{2}$ are the eigenvalues of $L ; \vec{u}_{l}$ are the left singular vectors of $M$ and eigenvectors of $L$. Equivalently, $\left(u_{a, l}\right)_{a, l=1}^{3 N(N-1) / 2}$ is a real orthogonal matrix, defining a family of operators

$$
\hat{Q}_{l} \equiv \sum_{a=1}^{3 N(N-1) / 2} u_{a, l} \hat{O}_{a}, \quad l=1, \ldots, 3 N(N-1) / 2
$$

which are also orthonormal:

$$
\frac{1}{\mathcal{D}} \operatorname{Tr}\left[\hat{Q}_{l}^{\dagger} \hat{Q}_{k}\right]=\delta_{l k} .
$$

As mentioned above, $Q_{l}$ is an integral of motion if and only if $\sigma_{l}=0$. Furthermore, for small $\sigma_{l}>0$, we consider $Q_{l}$ to be approximately conserved and call it a "slow mode." The rationale for this terminology comes from the identity

$$
\overline{\left\langle Q_{l}(t)\right\rangle^{2}}-\overline{\left\langle Q_{l}(t)\right\rangle^{2}}=\sigma_{l}^{2},
$$

which means that the singular value $\sigma_{l}$ is the standard deviation of the expectation value of $Q_{l}$ over the time interval $[0, T]$. A small $\sigma_{l}$ entails that $\left\langle Q_{l}(t)\right\rangle$ exhibits small fluctuations around its time-average value.

To summarize, we propose the following procedure: Compute the time series matrix $M$, perform a SVD decomposition on $M$, analyze its singular values, and identify the possible IOMs and slow modes. In the next two sections, we use this method to characterize the behavior of the model along the $S=1 / 2$ and $S \rightarrow \infty$ lines in the phase diagram of Fig. 1, for anisotropies $\Delta \neq 0,1$. In Sec. VI, we numerically simulate the time evolution for the quantum spin- $1 / 2$ model, and we further characterize the resulting slow modes by measuring their temporal autocorrelation functions. In Sec. VII, we simulate the dynamics of the model (1.2) describing classical spin d.o.f. and, upon slightly modifying the above method, we extract the behavior of the autocorrelation functions directly from the singular values.

\section{INTEGRABILITY* FOR $S=\frac{1}{2}$}

\section{A. Identifying integrals of motion}

We now focus on the spin- $1 / 2$ system with up to $N=14$ sites and implement the technique proposed above. We initialize the system in a random product state [61] $|\Phi\rangle$ and numerically compute the time evolution of the wave function with the Hamiltonian (1.2) via exact diagonalization. The random fields $J_{i}$ are sampled from the normal distribution $\mathcal{N}\left(0, J^{2}\right)$, and we set $J^{2}=1$. We have checked that we obtain similar results for other distributions with zero mean and unit variance. We then record the expectation values of all the operators $\hat{O}_{a}$ defined in Eq. (5.1) and construct the time series matrix $M_{a, n}$ [defined in Eq. (5.4)] at each discrete time $t_{n}=n \delta t$ with $\delta t=1 J^{-2}$, integer $n$, and up to a maximal time $T=10^{3} \mathrm{~J}^{-2}$.

Figure 2 presents results for the singular values of $M$ obtained for two values of $\Delta$ in a fixed disorder realization. As expected, at $\Delta=1$, we find $N+1$ vanishing singular values, in agreement with the analysis of Sec. IV. All other singular values lie above a gap of about 0.01 , indicating that 


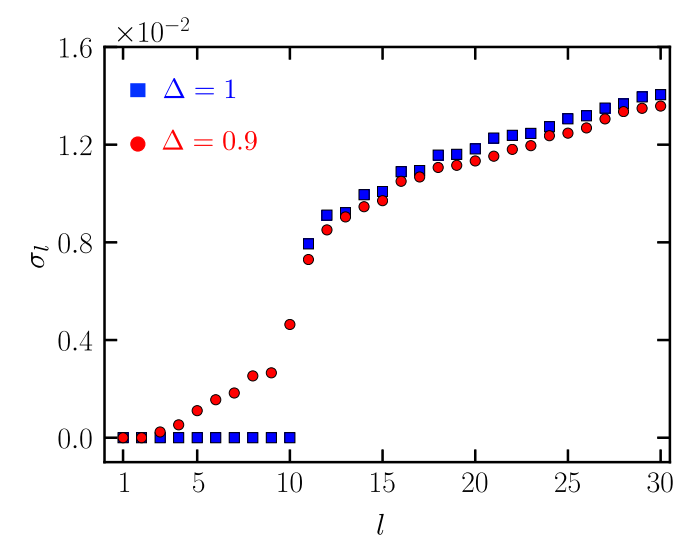

FIG. 2. Scatter plot of the smallest 30 of the $3 N(N-1) / 2$ singular values $\sigma_{l}$ at $N=9$ for $\Delta=1$ (blue squares) and $\Delta=0.9$ (red circles) in a fixed disorder realization of $\left\{J_{i}\right\}$. At $\Delta=1$, we see $N+1$ zeros corresponding to the $N+1$ conserved charges that can be written as a sum over bilinear operators; these zeros are separated from the rest of the singular values by a "spectral" gap. At $\Delta=0.9$, we see two zeros corresponding to the conservation of $H$ and $\left(S_{\text {tot }}^{z}\right)^{2}$. We also see the lift-off of $N-2$ singular values corresponding to the previously conserved bilinear charges at $\Delta=1$. Note that they, too, are separated from the rest by a "spectral" gap.

there are no other 2-local integrals of motion beyond those identified in Sec. IV.

The results at $\Delta=0.9$, slightly away from the integrable point, are markedly different. We find only two exactly vanishing singular values corresponding to the space spanned by the two obvious integrals of motion, $H$ and $\left(S_{\text {tot }}^{z}\right)^{2}$. This behavior persists on the entire open segment $\Delta \in(0,1)$, showing unambiguously that there are no other purely bilinear integrals of motion in this range. Nonetheless, we see that the remaining set of $N-2$ nontrivial IOMs at $\Delta=1$ are transformed, upon moving to the point $\Delta=0.9$, into left singular vectors with nonzero yet small singular values. It stands to reason that these small singular values correspond to operators that exhibit a slow decay because the system is close to the $\Delta=1$ integrable point. We now test this hypothesis by directly examining the decay of these putative "slow modes."

\section{B. Characterizing the slow operators}

We have seen that the nontrivial IOMs at the points $\Delta=0$ and $\Delta=1$ transform into a set of $N-2$ "slow operators," indicated by small singular values, away from those two points. Let us examine the dynamics of these presumed slow modes. Their decay can be studied by numerically computing the autocorrelation functions

$$
G_{l}(t)=\frac{1}{\mathcal{D}} \operatorname{Tr}\left[\hat{Q}_{l}(t) \hat{Q}_{l}(0)\right],
$$

where the normalization $\mathcal{D}=(2 S+1)^{N}$ ensures that $G_{l}(0)=1$. For conserved modes, we expect the

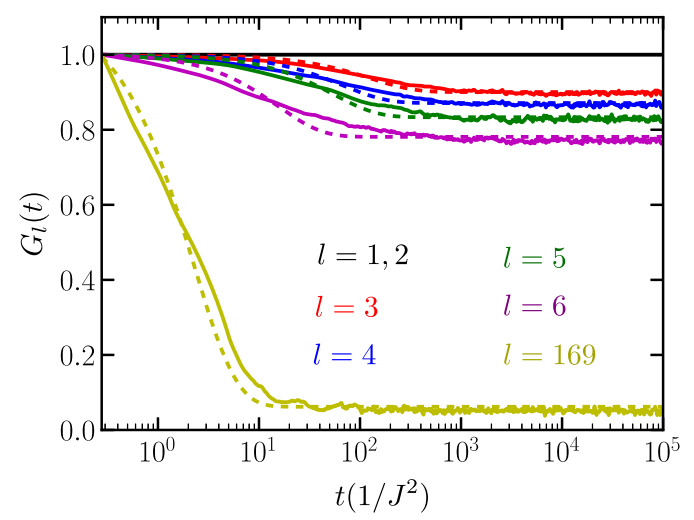

FIG. 3. Plot of the autocorrelation function $G_{l}(t)$ in a given disorder realization for $N=13$ spins at $\Delta=0.75$. The solid curves represent the numerically computed $G_{l}(t)$ : The black curve corresponds to either of the two exactly conserved bilinear quantities; the red, blue, green, and magenta curves correspond to the next four modes (arranged by increasing singular value); the yellow curve corresponds to a mode in the middle of the singular value "spectrum." The dashed curves represent fits of the form $\tilde{G}_{l}(t)=\zeta_{l} \exp \left(-t / \tau_{l}\right)+g_{l}$ through the data.

autocorrelation function to remain fixed at $G_{l}(t)=1$ for all time. For generic nonconserved operators, we expect $G_{l}(t)$ to decay to values near zero as these modes thermalize.

An example of the results for a system with $N=13$ sites and $\Delta=0.75$ is shown in Fig. 3. We see that the correlation functions related to the two zero singular values, $G_{1}(t)$ and $G_{2}(t)$, are perfectly nondecaying, as they must be. Also as expected, the correlation functions $G_{l}(t)$ associated with

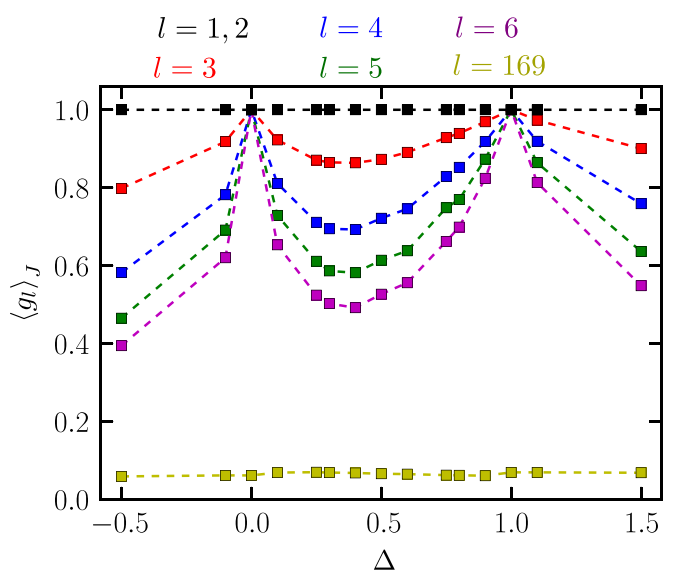

FIG. 4. Plot of the plateau values $\left\langle g_{l}\right\rangle_{J}=\left\langle G_{l}(t \rightarrow \infty)\right\rangle_{J}$ as a function of the anisotropy $\Delta$ for $N=11$ spins $(S=1 / 2)$. The brackets $\langle\ldots\rangle_{J}$ denote an average over 2000 disorder realizations for the $\left\{J_{i}\right\}$. Different colors correspond to different modes: Black corresponds to the two lowest and exactly conserved modes; red, blue, green, and magenta correspond to the next four modes; yellow corresponds to a mode in the middle of the singular-value spectrum. We find no strong dependence on the system size $N$ : See Fig. 5 for a plot of the plateau value $\left\langle g_{l}\right\rangle_{J}$ as a function of the system size $N$ for the $l=3$ (red) mode at $\Delta=0.5$. 

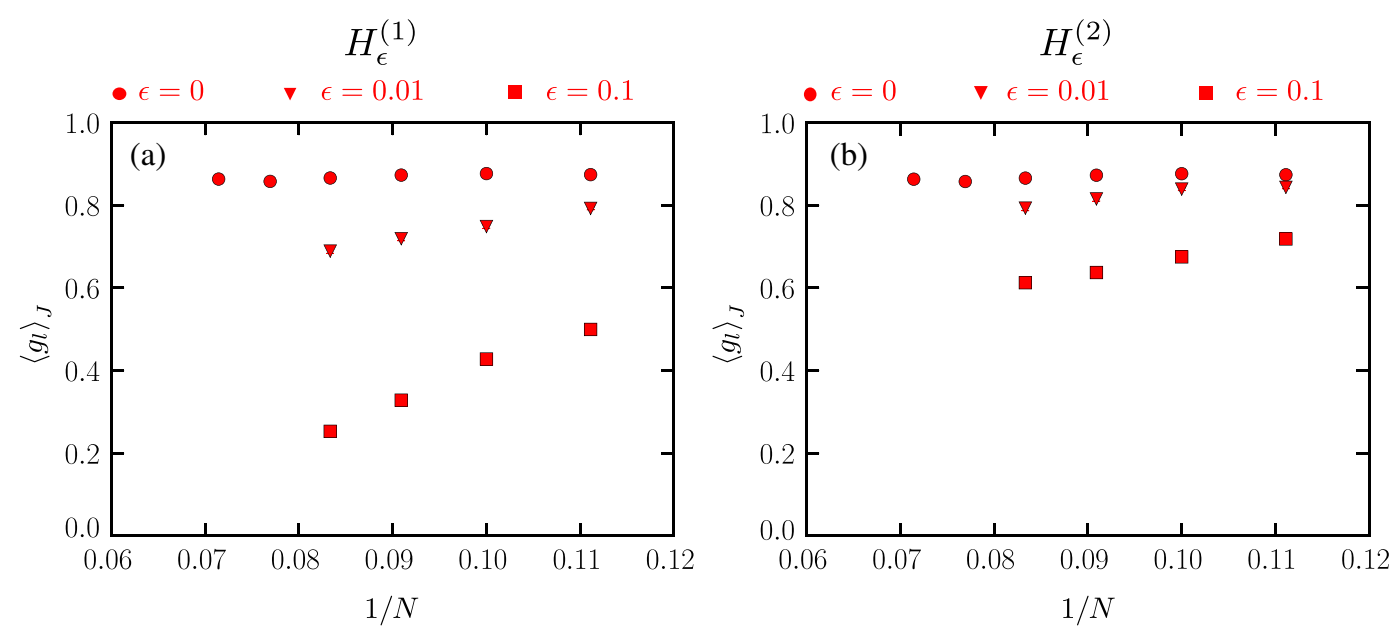

FIG. 5. Plot of the disorder-averaged plateau values $\left\langle g_{l}\right\rangle_{J}=\left\langle G_{l}(t \rightarrow \infty)\right\rangle_{J}$ as a function of the system size $N$ for the $l=3$ mode, i.e., the lowest mode that is not exactly conserved (corresponding to the red markers in Fig. 4) at $\Delta=0.5$. The different markers correspond to various strengths $\epsilon$ of the perturbations $H_{\epsilon}^{(1)}$ (left panel) and $H_{\epsilon}^{(2)}$ (right panel) from Eqs. (6.3) and (6.4), respectively: The round markers correspond to the unperturbed Hamiltonian $H(1.2)$; the triangular and square markers correspond to $\epsilon=0.01$ and $\epsilon=0.1$, respectively. The error bars related to disorder averaging $\langle\ldots\rangle_{J}$ are included, but they are smaller than the size of the markers. We see that the plateau value for the unperturbed $H$ is independent of the system size. Conversely, upon adding even a small perturbation $\epsilon \ll 1$, the plateau value decreases with $N$, suggesting that the autocorrelation function $G_{l}(t \rightarrow \infty)$ vanishes for a thermodynamic system $(N \rightarrow \infty)$.

the small nonvanishing singular values $(3 \leq l \leq N)$ show a slow initial decay. However, the surprise is that, at very long times, these correlation functions saturate to a nonvanishing and rather appreciable value $g_{l}$. Figure 4 shows that this phenomenon persists when varying $\Delta$ on the segment $[-0.5,1.5]$. Moreover, we find no significant size dependence of the saturation value $g_{l}$, as shown in Fig. 5(a). We have also checked that the large plateau values are not due to the overlap between the slow modes $\hat{Q}_{l}$ with higher powers of the known conservation laws $\hat{H}$ and $\hat{S}_{\text {tot }}^{z}$, such as $\hat{H}^{2}, \hat{H}^{3}, \ldots$, nor with projectors to energy eigenstates [35]. In contradistinction, the operators corresponding to higher singular values $(l \gg N)$ decay to a vanishing, or very small, saturation value [35].

Altogether, in addition to the obvious bilinear IOMs, $H$ and $\left(S_{\text {tot }}^{\mathrm{z}}\right)^{2}$, we find $N-2$ operators whose correlation functions saturate to an appreciable nonvanishing value. This result suggests that the model remains integrable even away from the Gaudin-like points $\Delta=0$ and $\Delta=1$ : The bilinear integrals of motion are transformed into quasibilinear ones, which retain appreciable support in the space of 2-local operators. Based on the results shown in Fig. 4, we argue that this holds everywhere away from the integrable points, namely, in the regions $\{\Delta<0\},\{0<\Delta<1\}$, and $\{\Delta>1\}$. In general, we can write the new integrals of motion as bilinear operators dressed by a sum over higher, $2 n$-local terms:

$\hat{I}_{l}=Z_{l} \hat{Q}_{l}+\sum_{n>1} \sum_{i_{1}, \ldots, i_{2 n}} \sum_{\alpha_{1}, \ldots, \alpha_{2 n}} K_{i_{1} \ldots i_{2 n}}^{\alpha_{1} \ldots \alpha_{2 n}} \hat{S}_{i_{1}}^{\alpha_{1}} \hat{S}_{i_{2}}^{\alpha_{2}} \ldots \hat{S}_{i_{2 n}}^{\alpha_{2 n}}$, where $Z_{l}$ is the weight of the integral of motion $I_{l}$ on 2-local operators. The saturation value of the autocorrelation function of $\hat{Q}_{l}$ that we plot in Fig. 4 is, essentially, $g_{l} \sim\left|Z_{l}\right|^{2}$. It would be interesting to further characterize how the coefficients $K_{i_{1} \ldots i_{2 n}}^{\alpha_{1} \ldots \alpha_{2 n}}$, which encode the overlap of the IOMs with the different $2 n$-body spin operators, decay with increasing $n$. We leave this for future work.

The structure of the integrals of motion (6.2) is, in some ways, reminiscent of the local integrals of motion in the MBL state $[49,50,62]$. The latter is characterized by quasilocal integrals of motion $\tau_{i}^{z}$ that are adiabatically connected to the microscopic d.o.f. $\sigma_{i}^{z}$. As in our case, the LIOMs are dressed versions of the microscopic bits with weight on higher $n$-body operators decaying exponentially with $n$. There are, however, crucial differences from MBL. The integrals of motion in our case are not local but rather extensive sums of bilocal operators. Hence, the IOMs of the all-to-all spin model do not facilitate a direct-product partition of the Hilbert space into single qubit spaces. Additionally, the integrability we observe does not depend on strong disorder-in fact, we found that its signatures are more pronounced as the couplings becomes more uniform, namely, as $\operatorname{std}\left(J_{i}\right) \lesssim \overline{J_{i}}$.

Lastly, we also find signatures of integrability in the spectrum of $H$ : The level statistics are almost perfectly Poissonian at $\Delta=0,1$ and close to Poisson (although not exactly) at intermediate $\Delta$ [35]. Nonetheless, for $0<$ $\Delta<1$, we find many level crossings, and the violation of the Wigner-von Neumann noncrossing rule represents further evidence of integrability despite the fact that there 
seems to be some degree of correlation between the energy levels [63-65].

\section{Perturbing away from Integrability*}

After establishing the existence of a novel integrable structure for the spin-1/2 model, characterized by quasi2-local IOMs, it is natural to investigate its robustness to perturbations away from the class of models (1.2) with separable disorder. This question is relevant from a theoretical point of view but also from a practical, experimental perspective.

A natural perturbation to test in this context is one that adds a nonseparable, SY-like contribution to the interaction. Specifically, we add the term

$$
H_{\epsilon}^{(1)}=\frac{\epsilon}{2 S \sqrt{N}} \sum_{i, j=1}^{N} V_{i j}\left[S_{i}^{x} S_{j}^{x}+S_{i}^{y} S_{j}^{y}+\Delta S_{i}^{z} S_{j}^{z}\right],
$$

where the elements $V_{i j}$ are also sampled from a normal distribution $\mathcal{N}(0,1)$.

We explicitly check that at $\epsilon>0$ and $\Delta=1$ for $H+H_{\epsilon}^{(1)}$, there are only four zero singular values corresponding to exactly conserved and linearly independent 2local quantities: the Hamiltonian, $S_{\text {tot }}^{2},\left(S_{\text {tot }}^{x}\right)^{2}$, and $\left(S_{\text {tot }}^{y}\right)^{2}$. At intermediate $0<\Delta<1$, there are only two vanishing singular values corresponding to $H+H_{\epsilon}^{(1)}$ and $\left(S_{\text {tot }}^{z}\right)^{2}$. Second, we verify that the lowest bilinear modes that are not exactly conserved (i.e., either the $l=3$ one at $0<\Delta<1$ or the $l=5$ one at $\Delta=1$ ) decay to smaller plateau values that decrease as we increase the system size $N$, as shown in Fig. 5(a). This suggests that a perturbation $H_{\epsilon}^{(1)}$, even at $\epsilon \ll 1$, can spoil the integrability for a large system $N \gg 1$.

Another type of perturbation that arises naturally in the experimental setup, due to the driving field, is represented by random stray magnetic fields along the $z$ axis:

$$
H_{\epsilon}^{(2)}=\epsilon \sum_{i=1}^{N} h_{i} S_{i}^{z}
$$

where the fields $h_{i}$ are also sampled from $\mathcal{N}(0,1)$. Note that $H+H_{\epsilon}^{(2)}$ has a single zero singular value corresponding to $\left(S_{\text {tot }}^{z}\right)^{2}$ for all $\Delta$ due to the fact that the full Hamiltonian is no longer purely bilinear and that $H_{\epsilon}^{(2)}$ breaks the SU(2) symmetry at $\Delta=1$. Aside from this effect, the behavior upon perturbing with $H_{\epsilon}^{(2)}$ is similar to that obtained by perturbing with $H_{\epsilon}^{(1)}$, as shown in Fig. 5(b).

Last, we consider the effect of adding the perturbation

$$
H^{(3)}=\frac{1}{S \sqrt{N}} \sum_{i} J_{i}^{2} S_{i}^{z}
$$

This additional term appears in the model

$$
\tilde{H}=\frac{1}{S \sqrt{N}} \sum_{i j} J_{i} J_{j}\left(S_{i}^{+} S_{j}^{-}+\Delta S_{i}^{z} S_{j}^{z}\right),
$$

which is similar to Eq. (1.2) but differs from it by the term $H^{(3)}$, arising due to the commutator $\left[S_{i}^{+}, S_{i}^{-}\right]$. As noted in Ref. [32], the model Eq. (6.6) is also experimentally accessible in a system of cold atoms interacting with cavity photons. It is clear that the perturbation $H^{(3)}$, having a $1 / \sqrt{N}$ normalization, is subextensive and will not matter in the thermodynamic limit. Moreover, we find that it does not qualitatively affect the integrability of our quantum model even for the small systems analyzed using exact diagonalization [35].

In sum, our numerical analysis of the response to perturbations indicates that the novel integrability of the spin- $1 / 2$ model (1.2) is not particularly robust to nonseparable interactions or stray magnetic fields. Nevertheless, in a finite-size system and at finite times (see Sec. VIII for more details), there are signatures of proximate integrability, as shown by the finite saturation values in Fig. 5 .

To recapitulate our study of the dynamical phase diagram in Fig. 1 thus far, we have found that the system is integrable along the following lines: at $\Delta=0,1$ for any value of the spin size $S$ (characterized by bilinear IOMs), and at $S=1 / 2$ for any $\Delta \neq 0,1$ (characterized by quasibilinear IOMs). The remaining line in the phase boundary of Fig. 1 corresponds to the classical, $S \rightarrow \infty$, limit of the model (1.2), which we now discuss.

\section{CHAOS FOR $S \rightarrow \infty$}

Since Gaudin-type integrability at $\Delta=0,1$ persists for all values of the spin size $S$, it is natural to ask whether the integrable* structure at $S=\frac{1}{2}$, presented in the previous section, also survives for larger values of $S$. Although it is numerically challenging to extend the exact diagonalization study of the previous section to intermediate $S$, the limit $S \rightarrow \infty$ leads to classical equations of motion that are amenable to numerical simulations.

These simulations allow us to analyze another boundary in the phase diagram, namely, the $S \rightarrow \infty$ line, where we find chaotic dynamics with a finite Lyapunov exponent, as explained in Sec. VII A. The presence of chaos in the infinite- $S$ limit clearly implies that the $S=\frac{1}{2}$ integrability* does not extend to all $S$, unlike the Gaudin-type integrability at $\Delta=0$ and 1 . Remnants of an integrable* structure can nevertheless be revealed by applying the SVD analysis of Sec. V to the classical dynamics, which we do in Sec. VII B. This technique reveals the presence of a large number of slow modes, which are known to occur classically in quasiintegrable systems, i.e., chaotic systems in the vicinity of integrable points. We characterize these slow modes in Sec. VII C.

\section{A. Classical chaos}

In the infinite- $S$ limit, the model (1.2) behaves as a classical system of coupled spin d.o.f. $S_{i}^{\alpha}$ on the unit sphere, whose Hamiltonian dynamics can be written in terms of Poisson brackets: 

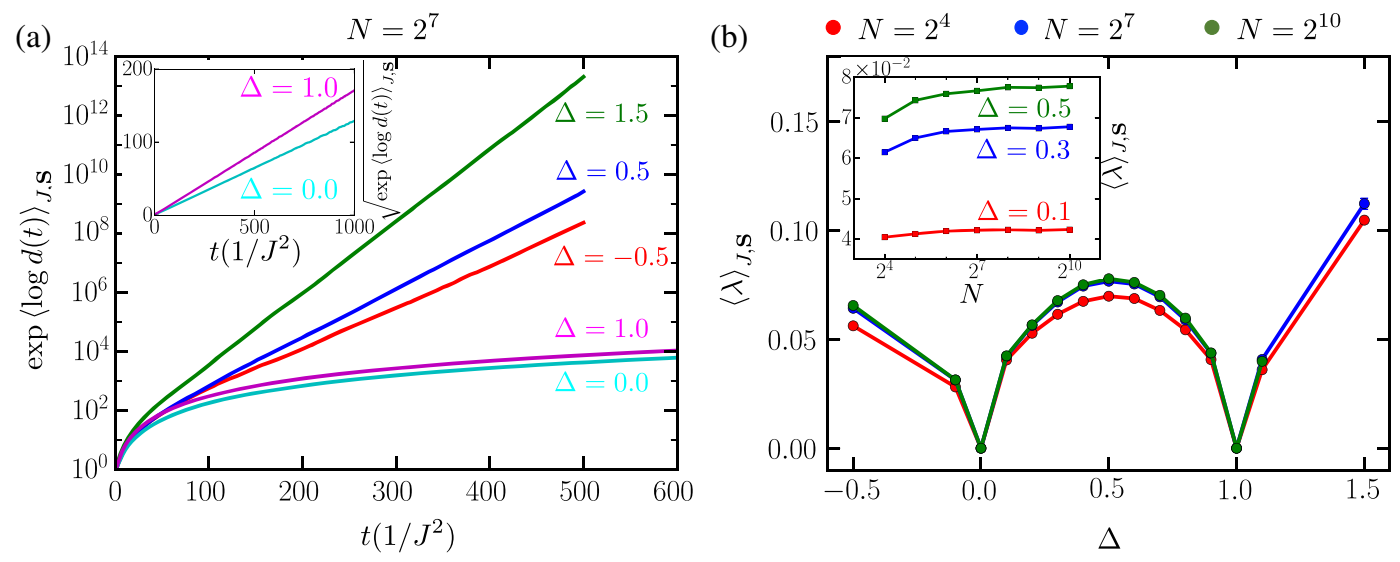

FIG. 6. (a) The sensitivity $d(t)$ from Eq. (7.7) geometrically averaged over $10^{4}$ disorder realizations $\left\{J_{i}\right\}$ and initial states $\{\mathbf{S}(0)\}$ as a function of time for a system of $N=128$ classical spins. After nonuniversal dynamics at early times, we find an exponential growth at later times for $\Delta=-0.5$ (red curve), $\Delta=0.5$ (blue curve), and $\Delta=1.5$ (green curve). (Inset) The square root of the same quantity, namely, $\sqrt{\exp \left(\langle\log d(t)\rangle_{J, S}\right)}$, at the two integrable points, $\Delta=0.0$ (cyan curve) and $\Delta=1.0$ (magenta curve). We obtain an almost perfect straight line, which indicates that $d(t) \sim t^{2}$, as expected for an integrable system. (b) Lyapunov exponent $\langle\lambda\rangle_{J, \mathbf{S}}$ averaged over $10^{5}$ disorder realizations and initial states as a function of the anisotropy $\Delta$ for different systems consisting of $N=16$ (red circles), $N=128$ (blue circles), and $N=1024$ (green circles) classical spins. (Inset) Disorder-averaged Lyapunov exponent $\langle\lambda\rangle_{J, \mathbf{S}}$ as a function of the system size $N$ for $\Delta=0.1$ (red squares), $\Delta=0.3$ (blue squares), and $\Delta=0.5$ (green squares).

$$
\frac{d S_{i}^{\alpha}}{d t}=\left\{S_{i}^{\alpha}, H\right\}
$$

where

$$
H=\frac{S}{2 \sqrt{N}} \sum_{i j} J_{i} J_{j}\left(S_{i}^{x} S_{j}^{x}+S_{i}^{y} S_{j}^{y}+\Delta S_{i}^{z} S_{j}^{z}\right) .
$$

For our numerical investigation, we sample the random fields $J_{i}$ from the uniform distribution $[-J, J]$ and set $J=1$ (we choose a bounded distribution to avoid large $J_{i}$ 's that could cause numerical instabilities). The classical spin variables $S_{i}^{\alpha}$ obey

$$
\left\{S_{i}^{\alpha}, S_{j}^{\beta}\right\}=\frac{1}{S} \delta_{i j} \varepsilon^{\alpha \beta \gamma} S_{i}^{\gamma} .
$$

We probe the infinite-temperature dynamics of this classical system by direct numerical simulation.

In order to study chaos, we use the standard tangent space method [66] to study the divergence of classical trajectories and measure the leading Lyapunov exponent. Let $\mathbf{S}(t)=\left(\mathbf{S}_{1}(t), \ldots, \mathbf{S}_{N}(t)\right)$ denote the $3 N$-dimensional vector describing the directions of all the spins at time $t$. We initialize the system in a random infinite-temperature state $\mathbf{S}(0)$, within which each spin points in a random direction, uniformly distributed on the unit sphere $S_{2}$. We also keep track of the trajectory of the deviation vector $\delta \mathbf{S}(t)$, which lives in the tangent space of $S_{2} \times \ldots \times S_{2}$ at the point $\mathbf{S}(t)$; we further set $\delta \mathbf{S}(0)$ such that $\delta \mathbf{S}_{i}(0) \perp \mathbf{S}_{i}(0)$ for all spins and $\|\delta \mathbf{S}(0)\|^{2}=\sum_{i, \alpha}\left(\delta S_{i}^{\alpha}\right)^{2}=1$.
If we define the local effective field

$$
\begin{aligned}
\mathbf{F}_{i} & =\left(F_{i}^{x}, F_{i}^{y}, F_{i}^{z}\right) \\
& =(1 / S)\left(-\partial H / \partial S_{i}^{x},-\partial H / \partial S_{i}^{y},-\partial H / \partial S_{i}^{z}\right),
\end{aligned}
$$

we see that the Hamilton equations of motion (7.1) can be written as

$$
\frac{d \mathbf{S}_{i}}{d t}=\mathbf{S}_{i} \times \mathbf{F}_{i}
$$

For our model (7.2), we have $F_{i}^{x, y}=\left(J_{i} / \sqrt{N}\right) \sum_{j} J_{j} S_{j}^{x, y}$ and $F_{i}^{z}=\Delta\left(J_{i} / \sqrt{N}\right) \sum_{j} J_{j} S_{j}^{z}$.

We immediately see that the variational equations of motion for the deviation vector $\delta \mathbf{S}$ can be written as

$$
\frac{d\left(\delta \mathbf{S}_{i}\right)}{d t}=\delta \mathbf{S}_{i} \times \mathbf{F}_{i}+\mathbf{S}_{i} \times \delta \mathbf{F}_{i},
$$

where $\delta F_{i}^{x, y}=\left(J_{i} / \sqrt{N}\right) \sum_{j} J_{j} \delta S_{j}^{x, y}$ and $\delta F_{i}^{z}=\Delta\left(J_{i} / \sqrt{N}\right)$ $\sum_{j} J_{j} \delta S_{j}^{z}$.

We numerically integrate the coupled differential equations (7.5) and (7.6) to find the trajectory $(\mathbf{S}(t), \delta \mathbf{S}(t))$ in the tangent bundle up until a time $T=500 \mathrm{~J}^{-2}$ in increments of $\delta t=1 J^{-2}$. We then compute the sensitivity, defined as $d(t)=\|\delta \mathbf{S}(t)\|^{2}$, or in full,

$$
d(t)=\sum_{i=1}^{N} \sum_{\alpha}\left[\delta S_{i}^{\alpha}(t)\right]^{2} .
$$


Note that $d(0)=1$ since we have normalized the initial deviation vector. For an integrable system, we expect $d(t)$ to exhibit a power-law dependence on time; the flow on invariant tori specified by the $N$ conservation laws is linear in time and, since we have defined the sensitivity as $\|\delta \mathbf{S}(t)\|^{2}$, we expect $d(t) \sim t^{2}$. In a chaotic system, $d(t)$ should increase exponentially with $t$. In Fig. 6(a), we average over disorder realizations $\left\{J_{i}\right\}$ and initial states $\{\mathbf{S}(0)\}$ to find $\exp \left(\langle\log d(t)\rangle_{J, \mathbf{S}}\right)$. We find that the classical system exhibits chaotic dynamics and an exponential divergence of trajectories in the regions $\{\Delta<0\}$, $\{0<\Delta<1\}$, and $\{\Delta>1\}$. We also find integrable dynamics and a power-law divergence of trajectories at the special points $\Delta=0,1$.

Moreover, using the multiplicative ergodic theorem, we can define the maximal Lyapunov exponent [66] as

$$
\lambda=\lim _{t \rightarrow \infty} \frac{2}{t} \log \frac{\|\delta \mathbf{S}(t)\|}{\|\delta \mathbf{S}(0)\|} .
$$

Using the normalization $\|\delta \mathbf{S}(0)\|=1$ and our definition of the sensitivity from Eq. (7.7), we see that

$$
\lambda=\lim _{t \rightarrow \infty} \frac{1}{t} \log d(t) .
$$

In practice, we compute the Lyapunov exponent by fitting a line $\lambda t+b$ through the late-time behavior of $\log d(t)$, as discussed in Ref. [67]. In Fig. 6(b), we plot the Lyapunov exponent $\langle\lambda\rangle_{J, S}$, averaged over disorder realizations $\left\{J_{i}\right\}$ and initial states $\{\mathbf{S}(0)\}$, as a function of the anisotropy $\Delta$ and find that the system exhibits the most chaotic behavior (largest Lyapunov exponent) at $\Delta=1.5$. Second, we find that $\langle\lambda\rangle_{J, \mathbf{S}}$ tends to a finite value for large system sizes $N$, as shown in the inset of Fig. 6(b).

\section{B. SVD analysis}

Although the presence of chaos in the classical dynamics excludes proper integrability in the infinite- $S$ limit, it does not rule out the possibility of quasi-integrability, whereby some operators have very slow decay. We investigate this possibility by applying the SVD analysis of Sec. V to the classical dynamics. This method allows us to determine the number of exactly conserved quantities, corresponding to zero singular values, but also to look for slow modes, corresponding to small but finite singular values.

As expected, we find an extensive number of conserved quantities at $\Delta=0,1$ and only two exactly conserved quantities, corresponding to the Hamiltonian $H$ and $\left(S_{\text {tot }}^{z}\right)^{2}$, for all other values of the anisotropy $\Delta$. This intermediate regime, however, exhibits a large number of slow modes, which will be discussed in the next section.

Since we are now working with a classical system, a few important distinctions ought to be made from our earlier quantum analysis. First, we consider a slightly enlarged collection of bilinear operators:

$$
O_{a}= \begin{cases}3 S_{i}^{\alpha} S_{j}^{\alpha} & a=(i, j, \alpha), i<j \\ c_{1}\left(S_{i}^{x} S_{i}^{x}-S_{i}^{y} S_{i}^{y}\right) & a=(i, i, 1) \\ c_{2}\left(3 S_{i}^{z} S_{i}^{z}-1\right) & a=(i, i, 2),\end{cases}
$$

where $c_{1}=\sqrt{15} / 2$ and $c_{2}=\sqrt{5} / 2$. As before, $a=(i, j, \alpha)$ is a composite index. In the classical case, we also include bilinears with $i=j$ (which would be trivial in the spin- $1 / 2$ case). Note that there are only two independent such bilinears for each $i$, and the spherical harmonics (with spin 1) provide an orthonormal basis. Indeed, it can be checked that the bilinears $O_{a}$ defined in Eq. (7.10) satisfy the orthonormality relation

$$
\left\langle O_{a} O_{b}\right\rangle_{\mathbf{S}} \equiv \int \prod_{i} \frac{D \mathbf{S}_{i}}{4 \pi} O_{a} O_{b}=\delta_{a b},
$$

where $\langle[\ldots]\rangle_{\mathbf{S}}$ denotes an average over the infinite-temperature ensemble, while the integral $\int D \mathbf{S}_{i}$ is over the unit sphere.

Second, while a single initial state is sufficient in the quantum SVD analysis, we have to consider an ensemble of initial states in the classical setting because a single classical trajectory cannot visit the whole phase space due to energy conservation (a linear superposition of configurations does not exist classically). Here, we consider the infinite-temperature ensemble; namely, we sample $S_{0}=\left\{\mathbf{S}_{1}(0), \ldots, \mathbf{S}_{N}(0)\right\}$ as independent random points on the unit sphere. We then time evolve with Eq. (7.1) for a total time $T$ and measure the expectation value of the bilinears $O_{a}\left(t_{n}, S_{0}\right)$ at discrete intervals $t_{n}=n \delta t \in[0, T]$. Repeating this process for a large number $\mathcal{N}$ of initial conditions $\left\{S_{0}\right\}$ in the infinite-temperature ensemble, we construct the following matrix, analogous to the one in Eq. (5.4):

$$
M_{a,\left(t, S_{0}\right)}=\left(O_{a}\left(t, S_{0}\right)-\overline{O_{a}}\left(S_{0}\right)\right),
$$

where $\overline{O_{a}}\left(S_{0}\right)=\int_{0}^{T}[(d t) / T] O_{a}\left(t, S_{0}\right)$ represents the time average over one trajectory. The number of rows indexed by $a$ is, according to Eq. (7.10), $3 N(N-1) / 2+2 N$. The columns are indexed by time $t \in[0, T]$ and initial conditions $S_{0}$-in practice, we discretize the time axis (with the time step $\left.\delta t=1 J^{-2}\right)$ and draw a large number $\left(10^{3}\right)$ of samples for $S_{0}$.

The singular-value decomposition of $M$ is equivalent to diagonalizing the real Hermitian matrix $L \equiv M M^{\dagger}$, which can be obtained by averaging over the initial conditions $S_{0}$ :

$$
L_{a, b}=\left\langle\int_{0}^{T} \frac{d t}{T} M_{a,\left(t_{n}, S_{0}\right)} M_{b,\left(t_{n}, S_{0}\right)}\right\rangle_{\mathbf{s}} .
$$

Note that the average $\langle\ldots\rangle_{\mathbf{S}}$ is with respect to $S_{0}$ in the infinite-temperature thermal ensemble and should not be confused with the quantum expectation values $\langle\ldots\rangle$ (i.e., 


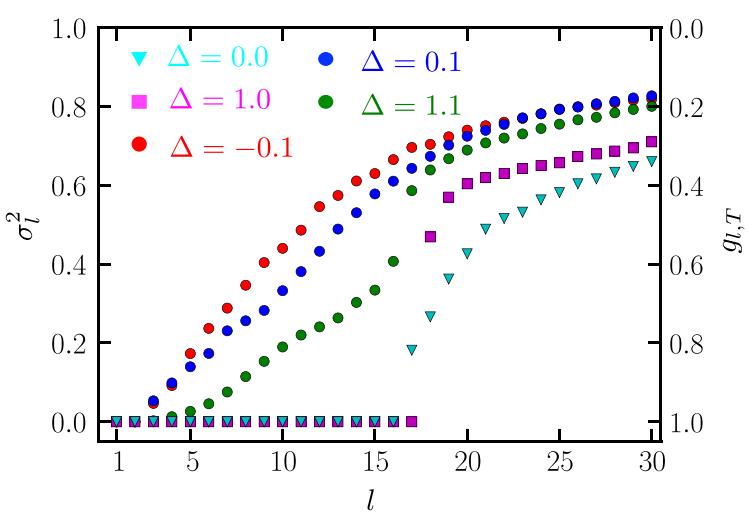

FIG. 7. Scatter plot of the smallest 30 squared singular values $\sigma_{l}^{2}$ and the plateau value $g_{l, T}$ from Eq. (7.17) for a classical model (7.2) of $N=16$ spins at different values of the anisotropy $\Delta$. We average $\sigma_{l}^{2}$ over $\mathcal{N}=10^{3}$ random initial conditions $\left\{S_{0}\right\}$ and 10 disorder realizations for the fields $\left\{J_{i}\right\}$, and we set $T=$ $8192 J^{-2}$. At the integrable points $\Delta=0$ (cyan triangles) and $\Delta=1$ (magenta squares), we see $N$ and $N+1$ zero singular values, respectively, corresponding to the conserved quantities that can be written as a sum over bilinear operators (7.10). These are separated from the rest of the singular values by a "spectral" gap. At $\Delta=-0.1$ (red circles), $\Delta=0.1$ (blue circles), and $\Delta=$ 1.1 (green circles), we see two precisely zero singular values corresponding to the conservation of $H$ and $\left(S_{\text {tot }}^{z}\right)^{2}$, along with the lift-off of the other $N-2$ singular values. For $\Delta=1.1$, the first $N$ singular values are also separated by a spectral gap from the rest. And, although for $\Delta=-0.1$ and $\Delta=0.1$ the spectral gap is not visible, one can still see a rounded "cusp" occurring around $l=N$-this cusp suggests that there still exist slow modes $Q_{l}$ for $l=3, \ldots, N$.

without a subscript) used in Secs. V and VI. Diagonalizing $L$ allows us to obtain the slow-mode operators $Q_{l}$, together with their corresponding eigenvalues $\sigma_{l}^{2}$. Similarly to Eq. (5.9), we have

$$
\sigma_{l}^{2}=\left\langle\overline{Q_{l}\left(t, S_{0}\right)^{2}}-\overline{Q_{l}\left(t, S_{0}\right)^{2}}\right\rangle_{\mathbf{s}} .
$$

In other words, $\sigma_{l}^{2}$ is equal to the variance, averaged over initial conditions $S_{0}$, of the fluctuations of $Q_{l}$ along a given trajectory.

The behavior of the singular values $\sigma_{l}$ (shown in Fig. 7) is similar, in several ways, to that obtained in Sec. VI A for the quantum spin- $1 / 2$ model [68]. At the first integrable point $\Delta=0$, we obtain $N$ zero singular values corresponding to the family of $N$ spin-bilinear conserved quantities $\tilde{G}^{(i)}$ from Eq. (4.10). At the second integrable point $\Delta=1$, we find $N+1$ zero singular values corresponding to the conserved quantities lying in the linear span of the $G^{(i)} \mathrm{s}$ from Eq. (4.8). Lastly, as shown in Fig. 7, away from these integrable points, i.e., for $\{\Delta<0\},\{0<\Delta<1\}$, and $\{\Delta>1\}$, we find two precisely zero singular values, corresponding to the two exactly conserved spin-bilinear quantities, $H$ and $\left(S_{\mathrm{tot}}^{z}\right)^{2}$. The small magnitude of the following singular values, for $l=3,4, \ldots$, signals the presence of slow modes, which will be studied in the next section.

\section{Decay of slow operators}

The SVD analysis of the previous section revealed a large number of operators with small singular values. In principle, we could characterize the thermalization (or lack thereof) of these operators $Q_{l}$ using, in analogy to the quantum case, a two-point correlation function

$$
G_{l}(t)=\left\langle Q_{l}(t) Q_{l}(0)\right\rangle_{\mathbf{S}},
$$

where $\langle\ldots\rangle_{\mathbf{S}}$, as before, designates an average over the $\mathcal{N}$ initial conditions $S_{0}$. As in the quantum case, the operators $Q_{l}$ are orthonormal such that $G_{l}(0)=1$ (as $\mathcal{N} \rightarrow \infty$ ). Yet, the accurate computation of $G_{l}(t)$ at long times is typically very demanding because it requires averaging an increasingly complex function in phase space.

Fortunately, in classical systems, the singular value $\sigma_{l}$ already informs us about the long-time plateau value of $G_{l}(t)$. This result can be seen from Eq. (7.14), which implies that

$$
\begin{aligned}
\sigma_{l}^{2} & =\left\langle\int_{0}^{T} \frac{d t}{T} Q_{l}(t)^{2}\right\rangle_{\mathbf{S}}-\left\langle\int_{0}^{T} \int_{0}^{T} \frac{d s}{T} \frac{d t}{T} Q_{l}(t) Q_{l}(s)\right\rangle_{\mathbf{S}} \\
& =1-\left\langle\int_{0}^{T} \int_{0}^{T} \frac{d s}{T} \frac{d t}{T} Q_{l}(t) Q_{l}(s)\right\rangle_{\mathbf{S}} \\
\sigma_{l}^{2} & =1-g_{l, T}, \quad g_{l, T} \equiv \int_{0}^{T} G_{l}(u) \frac{2(T-u) d u}{T^{2}}
\end{aligned}
$$

In the second line, we use the normalization $\left\langle Q_{l}(t)^{2}\right\rangle_{\mathbf{S}}=1$; in the third line, we perform a change of variables $u=|t-s|$ [recall that $G_{l}(u)=\left\langle Q_{l}(t) Q_{l}(t \pm u)\right\rangle_{\mathbf{S}}$ by the invariance of the infinite-temperature ensemble under time evolution]. Now, it is not hard to show that $g_{l, T}$ and $G_{l}$ have the same infinite-time limit (assuming such a limit exists for $G_{l}$ ):

$$
\lim _{u \rightarrow \infty} G_{l}(u)=g_{l} \Rightarrow \lim _{T \rightarrow \infty} g_{l, T}=g_{l} .
$$

Thus, $g_{l, T}$ is a finite-time proxy for $g_{l}$. In the infinite-time limit, the relation (7.16) becomes

$$
g_{l}=1-\left.\sigma_{l}^{2}\right|_{T \rightarrow \infty} .
$$

Using Eq. (7.16) or (7.17), this result allows us to infer the plateau values of slow modes from the data of Fig. 7. Unsurprisingly, the exactly conserved quantities have $g_{l}=1$. Away from the integrable points at $\Delta=0$, 1 , we find that the slowest nonconserved modes, corresponding to $l=3,4, \ldots$, (the distinction between the slow modes and the rest is less sharp here than in the quantum case, and it is suggested by the rounded cusp around $l=N$ in Fig. 7), have a remarkably slow decay: The plateau values $g_{l, T}$ at a finite 
but large time $T=10^{4} \mathrm{~J}^{-2}$ are close to unity [69], comparable to their spin- $1 / 2$ counterparts.

In a future companion paper, we will demonstrate that any conserved operator in the $S=1 / 2$ quantum case is approximately conserved in the $S \rightarrow \infty$ classical model as well, up to $1 / N$ corrections in large systems. Therefore, the classical model is expected to display some signatures of integrability. In this section, we saw that such signatures cannot be found from the Lyapunov exponent but only from the relaxation of slow modes. This result is intriguing, but a similar phenomenon has previously been observed. Reference [48] showed that for certain systems near integrability (called quasi-integrable by the authors), the relaxation time of certain operators can be significantly longer than the finite Lyapunov time $\lambda^{-1}$. Given that our classical system is surrounded by integrable lines $\Delta=0,1$ and (arguably) $S=1 / 2$ in the $(\Delta, S)$ parameter plane (see Fig. 1), we conjecture that it is also quasi-integrable. From this perspective, the existence of slow modes is compatible with the finite classical Lyapunov exponent found in Sec. VII A.

\section{EXPERIMENTAL REALITIES}

We have provided analytical and numerical evidence for the rich dynamical phase diagram depicted in Fig. 1, including clear signatures of chaotic dynamics at large $S$ and $\Delta \neq 0,1$, along with signatures of integrability at the special points $\Delta=0,1$ for any $S$. Furthermore, we demonstrated signatures of a novel integrable* phase at $S=1 / 2$ for $\Delta \neq 0,1$. We now discuss prospects for observing these signatures in the laboratory. First, what should one measure to identify the chaotic and integrable regimes of the phase diagram? Second, given the inevitable presence of dissipation in realistic experiments, what are the requirements on cavity cooperativity to access the relevant timescales experimentally?

To identify integrals of motion, the SVD method of Sec. V can equivalently be implemented with experimental data. Using state-sensitive imaging of the atomic ensemble [21], one may immediately extract the bilinear spin correlation functions $\left\langle O_{a}(t)\right\rangle \propto\left\langle S_{i}^{\alpha}(t) S_{j}^{\alpha}(t)\right\rangle$ defined in Eq. (5.1). As each image is obtained from a destructive measurement, one must repeat the experiment many times to obtain statistics of the spin bilinears at a fixed time $t$ and then repeat this procedure for many time points $t$ to obtain the full matrix $M_{a, t}$. With this matrix in hand, one can then directly apply the singular-value decomposition performed above in Sec. V.

A caveat is that measurements of the spin bilinears can be affected by dissipation due to photon loss and atomic free-space scattering. Photon loss from the cavity mode causes a random walk in the orientation of the weighted collective spin $\mathcal{F}$ defined in Eq. (3.1). This effect is described by Lindblad operators

$$
\begin{gathered}
L_{ \pm}=\sqrt{\gamma / 2} \mathcal{F}_{ \pm}, \\
L_{z}=\sqrt{\Delta \gamma} \mathcal{F}_{z},
\end{gathered}
$$

where the decay rate [35] is given by

$$
\gamma=\frac{J^{2}}{S \sqrt{N}} \frac{\kappa}{\delta}
$$

The collective dissipation can be suppressed by increasing the detuning $\delta$ and compensating with increased drive strength, until limited by free-space scattering.

The effect of free-space scattering is to project or flip individual spins, as described by a set of Lindblad operators

$$
L_{n,\left(m, m^{\prime}\right)}=\sqrt{C_{m, m^{\prime}} \Gamma_{\mathrm{sc}}}|m\rangle\left\langle\left. m^{\prime}\right|_{n},\right.
$$

where $m$ or $m^{\prime}$ indicates the spin state of an individual atom indexed by $n$, and $C_{m, m^{\prime}}$ is an order-unity branching ratio. At large detuning, the scattering rate scales as

$$
\Gamma_{\mathrm{sc}} \sim \frac{J^{2}}{\eta S \sqrt{N}} \frac{\delta}{\kappa} .
$$

Comparing Eqs. (8.3) and (8.5), we see that the cooperativity $\eta$ will dictate an optimal detuning for minimizing the net effect of the two forms of dissipation, with higher cooperativity enabling increasingly coherent dynamics.

To determine the cooperativity required to observe the signatures of integrability, we first write down explicit equations of motion for the spin bilinears $\left\langle S_{i}^{\alpha}(t) S_{j}^{\alpha}(t)\right\rangle$ evolving under the influence of pure collective dissipation or pure single-atom decay, respectively [35]. We find that the spin bilinears decay exponentially at a rate $\Gamma_{\mathrm{sc}}$ due to free-space scattering and at a rate $\gamma$ due to photon loss from the cavity. Notably, the rate of spin relaxation due to photon loss is not superradiantly enhanced, thanks to the counterbalanced effects of the $L_{ \pm}$Lindblad operators. Thus, at weak to moderate cooperativity $\eta \lesssim 1$ and large detuning $\delta>\kappa$, free-space scattering dominates, and the bilinears decay on a timescale $\tau J^{2} \sim \sqrt{N} \eta S \kappa / \delta$. For strong coupling $\eta \gg 1$, where free-space scattering is suppressed relative to cavity decay, the total dissipation can be minimized at a detuning $\delta \sim \sqrt{\eta} \kappa$, leading to the decay of spin bilinears on a timescale $\tau J^{2} \sim \sqrt{N \eta} S$.

To compare the decay time $\tau$ with the characteristic timescales for observing the signatures of integrability, we refer to the time dependence of the autocorrelation functions $G_{l}(t)$ shown in Fig. 3. To observe the slow modes, a minimum requirement is to evolve the system for a time $t \gtrsim t^{*} \approx 10 \mathrm{~J}^{-2}$, which governs the rapid decay of all nonintegrable autocorrelation functions. This time can be reached even at $S=1 / 2$ in a strong-coupling cavity $\eta \sim 10$ with a system of $N=10^{3}$ sites or with weaker single-atom cooperativity at larger $S$. To observe the plateaus themselves, we must evolve the system for a significantly longer 
time, at least $t \approx 10^{3} J^{-2}$ according to Fig. 3, which places a more stringent requirement $\sqrt{N} \eta S \gtrsim 10^{3} \delta / \kappa$. This regime is challenging to access for $S=1 / 2$ but readily accessible with large- $S$ subensembles, e.g., at $\eta \gtrsim 1$ with $N=10^{2}$ sites each consisting of $S=10^{3}$ spin- 1 atoms.

Thus, current experiments are well positioned to explore the regime of mesoscopic spin $S$, in between the quantum $(S=1 / 2)$ and classical $(S \rightarrow \infty)$ limits. This will allow for testing the prediction that the plateaus in $G_{l}(t)$ calculated for spin $S=1 / 2$, indicating integrability across the full range $\{\Delta<0\},\{0<\Delta<1\}$, and $\{\Delta>1\}$, persist for larger spin $S$ up to $1 / N$ corrections (see Sec. VII C). Experiments with scalable spin size $S$ may furthermore shed light on the transition from quantum integrability to chaos in the classical limit, as signified by the positive Lyapunov exponent in Fig. 6.

The chaotic dynamics observed in the classical limit $S \rightarrow \infty$ can be studied experimentally via the hallmark of sensitivity to perturbations. Recent theoretical and experimental work has shown that such sensitivity is accessible in quantum systems by measuring out-of-time-order correlators (OTOCs) [1,34,70-75], which quantify the spread of operators in time via the commutator $C(t)=\langle[V(t)$, $\left.W(0)]^{2}\right\rangle$. The connection to classical chaos is made clear in the semiclassical limit: For operators $V=S_{i}^{z}, W=S_{j}^{z}$, one can show that, to lowest order in a $1 / S$ expansion, $C(t) \propto$ $\left(\partial S_{i}^{z}(t) / \partial \phi_{j}\right)^{2}$ for a small rotation $\phi_{j}$ at site $j$ about the $z$ axis [76]. Thus, semiclassically, the OTOC $C(t)$ measures the sensitivity of the coordinate $S_{i}^{z}(t)$ to changes in initial conditions $S_{j}^{z}(0)$ and may therefore be regarded as a quantum generalization of the classical sensitivity $d(t)$ defined in Sec. VII A.

One way to access out-of-time-order correlators experimentally is to "reverse the flow of time" by dynamically changing the sign of the Hamiltonian [34,73]. In the singlemode cavity system considered here [21], this sign reversal is achieved by switching the sign of the laser detuning $\delta$ in Eq. (3.7). The resilience of such time-reversal protocols to experimental imperfections, including dissipation, has been analyzed theoretically in Ref. [77].

To allow for probing chaos in the optical cavity system proposed here, the rates of collective dephasing and of decoherence via single-atom decay must be small compared with the Lyapunov exponent. We thus require $\lambda \tau \gg 1$, where $\tau$ is the characteristic decay time defined above. More specifically, given the Lyapunov exponents $\lambda \leq 0.08 J^{2}$ shown in Fig. 6, and the requirement of observing the system for several Lyapunov "decades" to clearly identify exponential growth [Fig. 6(a)], we would like to evolve the system for times $t \gtrsim 100 \mathrm{~J}^{-2}$, which are readily accessible in the large- $S$ regime that is of interest for approaching the classical limit.

Even in this regime, the light leaking from the cavity produces a continuous weak measurement of the collective spin $\mathcal{F}$ whose quantum backaction may have consequences for the dynamics. The interplay of measurement backaction with chaos in open quantum systems, while beyond the scope of the present work, is a subject of active inquiry $[78,79]$ and of fundamental importance for elucidating the quantum-to-classical transition [80]. The proposed experimental scheme, including the possibility of tuning the strength and form of coupling to the environment, opens new prospects for exploring this interplay.

\section{DISCUSSION}

We have studied a class of spin models with separable, all-to-all, random interactions and found a complex dynamical phase structure that depends on the spin size $S$ and the anisotropy $\Delta$ along the $z$ axis. We showed that our model at $\Delta=1$ is equivalent to the well-studied rational Gaudin model and that it exhibits special integrable dynamics for all values of $S$. We also proved and confirmed numerically that there exists another special point at $\Delta=0$ where the model is also integrable (in the same sense), regardless of the spin size. Surprisingly, we found compelling numerical evidence that the system at $S=1 / 2$ is integrable for any anisotropy $\Delta \notin\{0,1\}$. In contrast to the special points $\Delta=0,1$, the integrals of motion at other values of $\Delta$ are not purely spin bilinears and develop tails on $2 n$-body terms. We leave the detailed characterization of these dressing tails to future work. Lastly, we found that integrability away from $\Delta=0,1$ is a purely quantum phenomenon: By numerically solving the Hamilton equations of motion for the classical model $(S \rightarrow \infty)$, we showed that its dynamics is chaotic with a nonzero Lyapunov exponent and that there exist only two exactly conserved quantities, as opposed to the extensive family of conservation laws characterizing a classically integrable system. However, even in the classical regime, we find an extensive number of quasiconserved charges, whose decay time appears to diverge in the large- $N$ limit. A more thorough study of this regime will be given in future work.

Our analysis opens up several further lines of inquiry. First, since the Hamiltonian (1.2) at the special point $\Delta=1$ (and, presumably, at $\Delta=0$ as well $[40,41,43-45])$ possesses a quantum group structure, does the integrable* phase exhibit any algebraic structure? Is it possible to construct explicitly the dressed conserved quantities in terms of the model couplings?

Second, we have seen that even though the level statistics of the spin-1/2 system deviates from Wigner-Dyson statistics, exhibiting many level crossings (this holds also for the spin-1 system [35]), its classical counterpart is chaotic with a finite Lyapunov exponent. We note that this does not contradict the Berry-Tabor conjecture $[81,82]$, which applies to the semiclassical, large- $S$ regime. In fact, the same phenomenon is known to occur in integrable quantum spin chains, such as the anisotropic Heisenberg model (or $X X Z$ chain): Its Hamiltonian $\sum_{j}\left[S_{j}^{x} S_{j+1}^{x}+S_{j}^{y} S_{j+1}^{y}+\Delta S_{j}^{z} S_{j+1}^{z}\right]$ is quantum integrable only for $S=1 / 2$, while the semiclassical 
limit $S \rightarrow \infty$ is classically chaotic. Integrable higher-spin extensions of the anisotropic Heisenberg model have different Hamiltonians that are nontrivial to obtain [83]. We wonder whether our integrable* phase admits any such extensions, which might shed light on the quasi-integrability of our classical model.

Third, we have only characterized the boundaries of the phase diagram in Fig. 1. A straightforward and interesting next step would be to study the quantum-to-classical crossover by better understanding how classical chaos (and perhaps quasi-integrability) at $S \rightarrow \infty$ emerges from the integrable* regime at $S=1 / 2$.

In fact, this putative transition between (quantum) integrability and (semiclassical) chaos may also be probed experimentally. The model (1.2) can be implemented in a near-term experiment using atomic ensembles confined in a single-mode optical cavity. Doing so would allow for a systematic exploration of the rich physics contained in the dynamical phase diagram (Fig. 1). By changing the local atom density to increase the number of atoms in a given region of constant coupling to the cavity mode, the spin size $S$ can be varied from $S=1 / 2$ all the way to a semiclassical regime $S \gg 1$, which would enable the experiment to tune between quantum and classical dynamics. Meanwhile, changing the angle between the magnetic field defining the spins' $z$ axis and the axis of the optical cavity enables tuning of the anisotropy $\Delta$, allowing for systematic investigation of both the special points $\Delta=0,1$ and the regions $\{\Delta<0\},\{0<\Delta<1\}$, and $\{\Delta>1\}$.

Last, we emphasize that the SVD technique described in Sec. V can be applied directly to the experimental data, revealing the conserved quantities and slow modes. More broadly, we envision using this approach in studying a wider class of physical systems wherein the integrals of motion or their number are not a priori known.

In summary, the model (1.2) and its associated experimental setup represent a novel paradigmatic platform for studying integrability, chaos, and thermalization under closed many-body quantum dynamics.

\section{ACKNOWLEDGMENTS}

We would like to thank Romain Vasseur, Fabian Essler, Thomas Klein Kvorning, Daniel E. Parker, Emily J. Davis, and Avikar Periwal for fruitful conversations. This work was supported by the DOE Office of Science, Office of High Energy Physics (G. B.), Grant No. DE-SC0019380 (X. C., X.L.Q., M.S.S., and E. A.), the ERC synergy grant UQUAM (I. D. P., X.C., and E.A.), and the Emergent Phenomena in Quantum Systems initiative of the Gordon and Betty Moore Foundation (T. S.). The numerical computations were carried out on the Lawrencium cluster resource provided by the IT Division at the Lawrence Berkeley National Laboratory under the DOE Contract No. DEAC02-05CH11231, on the Sherlock computing cluster provided by Stanford University and the Stanford
Research Computing Center, and on the cluster of the Laboratoire de Physique Théorique et Modèles Statistiques (CNRS, Université Paris-Sud).

[1] J. Maldacena, S. H. Shenker, and D. Stanford, A Bound on Chaos, J. High Energy Phys. 8 (2016) 106.

[2] A. Kitaev, A Simple Model of Quantum Holography (2015), http://online.kitp.ucsb.edu/online/entangled15/kitaev/ (part 1) \& http://online.kitp.ucsb.edu/online/entangled15/ kitaev2/ (part 2).

[3] J. Maldacena and D. Stanford, Remarks on the SachdevYe-Kitaev Model, Phys. Rev. D 94, 106002 (2016).

[4] S. Sachdev, Bekenstein-Hawking Entropy and Strange Metals, Phys. Rev. X 5, 041025 (2015).

[5] K. Jensen, Chaos in $\mathrm{AdS}_{2}$ Holography, Phys. Rev. Lett. 117, 111601 (2016).

[6] D. Stanford and E. Witten, Fermionic Localization of the Schwarzian Theory, J. High Energy Phys. 10 (2017) 008.

[7] S. Sachdev and J. Ye, Gapless Spin-Fluid Ground State in a Random Quantum Heisenberg Magnet, Phys. Rev. Lett. 70, 3339 (1993).

[8] A. T. Black, H. W. Chan, and V. Vuletić, Observation of Collective Friction Forces due to Spatial Self-Organization of Atoms: From Rayleigh to Bragg Scattering, Phys. Rev. Lett. 91, 203001 (2003).

[9] J. Majer, J. M. Chow, J. M. Gambetta, J. Koch, B. R. Johnson, J. A. Schreier, L. Frunzio, D. I. Schuster, A. A. Houck, A. Wallraff, A. Blais, M. H. Devoret, S. M. Girvin, and R. J. Schoelkopf, Coupling Superconducting Qubits via a Cavity Bus, Nature (London) 449, 443 (2007).

[10] I. D. Leroux, M. H. Schleier-Smith, and V. Vuletić, Implementation of Cavity Squeezing of a Collective Atomic Spin, Phys. Rev. Lett. 104, 073602 (2010).

[11] A. F. van Loo, A. Fedorov, K. Lalumière, B. C. Sanders, A. Blais, and A. Wallraff, Photon-Mediated Interactions Between Distant Artificial Atoms, Science 342, 1494 (2013).

[12] G. Barontini, L. Hohmann, F. Haas, J. Estève, and J. Reichel, Deterministic Generation of Multiparticle Entanglement by Quantum Zeno Dynamics, Science 349, 1317 (2015).

[13] O. Hosten, R. Krishnakumar, N. J. Engelsen, and M. A. Kasevich, Quantum Phase Magnification, Science 352, 1552 (2016).

[14] A. J. Kollár, A. T. Papageorge, V. D. Vaidya, Y. Guo, J. Keeling, and B. L. Lev, Supermode-Density-Wave-Polariton Condensation with a Bose-Einstein Condensate in a Multimode Cavity, Nat. Commun. 8, 14386 (2017).

[15] J. Léonard, A. Morales, P. Zupancic, T. Esslinger, and T. Donner, Supersolid Formation in a Quantum Gas Breaking a Continuous Translational Symmetry, Nature (London) 543, 87 (2017).

[16] M. A. Norcia, R. J. Lewis-Swan, J. R. K. Cline, B. Zhu, A. M. Rey, and J. K. Thompson, Cavity-Mediated Collective Spin-Exchange Interactions in a Strontium Superradiant Laser, Science 361, 259 (2018).

[17] R. M. Kroeze, Y. Guo, V. D. Vaidya, J. Keeling, and B. L. Lev, Spinor Self-Ordering of a Quantum Gas in a Cavity, Phys. Rev. Lett. 121, 163601 (2018). 
[18] M. Landini, N. Dogra, K. Kroeger, L. Hruby, T. Donner, and T. Esslinger, Formation of a Spin Texture in a Quantum Gas Coupled to a Cavity, Phys. Rev. Lett. 120, 223602 (2018).

[19] J. Kohler, J. A. Gerber, E. Dowd, and D. M. Stamper-Kurn, Negative-Mass Instability of the Spin and Motion of an Atomic Gas Driven by Optical Cavity Backaction, Phys. Rev. Lett. 120, 013601 (2018).

[20] Y. Guo, R. M. Kroeze, V. Vaidya, J. Keeling, and B. L. Lev, Sign-Changing Photon-Mediated Atom Interactions in Multimode Cavity QED, Phys. Rev. Lett. 122, 193601 (2019).

[21] E. J. Davis, G. Bentsen, L. Homeier, T. Li, and M. H. Schleier-Smith, Photon-Mediated Spin-Exchange Dynamics of Spin-1 Atoms, Phys. Rev. Lett. 122, 010405 (2019).

[22] B. Braverman, A. Kawasaki, E. Pedrozo-Peafiel, S. Colombo, C. Shu, Z. Li, E. Mendez, M. Yamoah, L. Salvi, D. Akamatsu, Y. Xiao, and V. Vuletić, Near-Unitary Spin Squeezing in $171 \mathrm{Yb}$, Phys. Rev. Lett. 122, 223203 (2019).

[23] A. André, L.-M. Duan, and M. D. Lukin,Coherent Atom Interactions Mediated by Dark-State Polaritons, Phys. Rev. Lett. 88, 243602 (2002).

[24] A. S. Sørensen and K. Mølmer, Entangling Atoms in Bad Cavities, Phys. Rev. A 66, 022314 (2002).

[25] M. H. Schleier-Smith, I. D. Leroux, and V. Vuletić, Squeezing the Collective Spin of a Dilute Atomic Ensemble by Cavity Feedback, Phys. Rev. A 81, 021804(R) (2010).

[26] S. Gopalakrishnan, B. L. Lev, and P. M. Goldbart, Frustration and Glassiness in Spin Models with Cavity-Mediated Interactions, Phys. Rev. Lett. 107, 277201 (2011).

[27] C.-L. Hung, A. González-Tudela, J. I. Cirac, and H. J. Kimble, Quantum Spin Dynamics with Pairwise-Tunable, Long-Range Interactions, Proc. Natl. Acad. Sci. U.S.A. 113, E4946 (2016).

[28] S. J. Masson, M. D. Barrett, and S. Parkins, Cavity QED Engineering of Spin Dynamics and Squeezing in a Spinor Gas, Phys. Rev. Lett. 119, 213601 (2017).

[29] F. Mivehvar, F. Piazza, and H. Ritsch, Disorder-Driven Density and Spin Self-Ordering of a Bose-Einstein Condensate in a Cavity, Phys. Rev. Lett. 119, 063602 (2017).

[30] E. Colella, R. Citro, M. Barsanti, D. Rossini, and M.-L. Chiofalo, Quantum Phases of Spinful Fermi Gases in Optical Cavities, Phys. Rev. B 97, 134502 (2018).

[31] F. Mivehvar, H. Ritsch, and F. Piazza, Cavity-QuantumElectrodynamical Toolbox for Quantum Magnetism, Phys. Rev. Lett. 122, 113603 (2019).

[32] J. Marino and A. M. Rey, A Cavity-QED Simulator of Slow and Fast Scrambling, Phys. Rev. A 99, 051803 (2019).

[33] P. Strack and S. Sachdev, Dicke Quantum Spin Glass of Atoms and Photons, Phys. Rev. Lett. 107, 277202 (2011).

[34] B. Swingle, G. Bentsen, M. Schleier-Smith, and P. Hayden, Measuring the Scrambling of Quantum Information, Phys. Rev. A 94, 040302(R) (2016).

[35] See Supplemental Material at http://link.aps.org/ supplemental/10.1103/PhysRevX.9.041011 for details, which includes a derivation of the effective spin Hamiltonian, analysis of realistic dissipation, a discussion of the appropriate normalization of the Hamiltonian, analysis of possible overlaps between the dressed LIOMs and trivial conserved quantities or energy eigenstates, and numerical computation of level statistics.

[36] M. Gaudin,Diagonalisation d'une Classe d'Hamiltoniens de Spin, J. Phys. France 37, 1087 (1976).

[37] E. K. Sklyanin, Separation of Variables in the Gaudin Model, J. Sov. Math. 47, 2473 (1989).

[38] A. Yu Kitaev, A. Shen, and M. N. Vyalyi, Classical and Quantum Computation, Vol. 47 (Graduate Studies in Mathematics, American Mathematical Society, 2002).

[39] D. Aharonov and T. Naveh, Quantum NP-A Survey, arXiv: quant-ph/0210077.

[40] A. B. Balantekin, T. Dereli, and Y. Pehlivan, Solutions of the Gaudin Equation and Gaudin Algebras, J. Phys. A 38, 5697 (2005).

[41] T. Skrypnyk, New Integrable Gaudin-type Systems and Classical r-Matrices and Quasigraded Lie Algebras, Phys. Lett. A 334, 390 (2005).

[42] J. R. Schmidt, Singular Point Analysis of the Gaudin Equations, Can. J. Phys. 86, 783 (2008).

[43] T. Skrypnyk, Spin Chains in Magnetic Field, Non-SkewSymmetric Classical r-Matrices and BCS-type Integrable Systems, Nucl. Phys. B806, 504 (2009).

[44] T. Skrypnyk, Non-Skew-Symmetric Classical r-Matrices, Algebraic Bethe Ansatz, and Bardeen-Cooper-SchriefferType Integrable Systems, J. Math. Phys. (N.Y.) 50, 033504 (2009).

[45] T. Skrypnyk, Non-Skew-Symmetric Classical r-Matrices and Integrable Cases of the Reduced BCS Model, J. Phys. A 42, 472004 (2009).

[46] I. Lukyanenko, P. S. Isaac, and J. Links, An Integrable Case of the $p+i p$ Pairing Hamiltonian Interacting with Its Environment, J. Phys. A 49, 084001 (2016).

[47] P. W. Claeys, C. Dimo, S. De Baerdemacker, and A. Faribault, Integrable Spin-1/2 Richardson-Gaudin XYZ Models in an Arbitrary Magnetic Field, J. Phys. A 52, 08LT01 (2019).

[48] T. Goldfriend and J. Kurchan, Equilibration of Quasiintegrable Systems, Phys. Rev. E 99, 022146 (2019).

[49] R. Vosk and E. Altman, Many-Body Localization in One Dimension as a Dynamical Renormalization Group Fixed Point, Phys. Rev. Lett. 110, 067204 (2013).

[50] M. Serbyn, Z. Papić, and D. A. Abanin, Local Conservation Laws and the Structure of the Many-Body Localized States, Phys. Rev. Lett. 111, 127201 (2013).

[51] D. A. Huse, R. Nandkishore, and V. Oganesyan, Phenomenology of Fully Many-Body-Localized Systems, Phys. Rev. B 90, 174202 (2014).

[52] A. Chandran, I. H. Kim, G. Vidal, and D. A. Abanin, Constructing Local Integrals of Motion in the Many-Body Localized Phase, Phys. Rev. B 91, 085425 (2015).

[53] V. Ros, M. Mller, and A. Scardicchio, Integrals of Motion in the Many-Body Localized Phase, Nucl. Phys. B891, 420 (2015).

[54] For a comprehensive discussion of the subtleties involved in achieving a generally valid definition of quantum integrability, see Ref. [55].

[55] J.-S. Caux and J. Mossel, Remarks on the Notion of Quantum Integrability, J. Stat. Mech. (2011) P02023 (2011).

[56] X. Zotos, F. Naef, and P. Prelovsek, Transport and Conservation Laws, Phys. Rev. B 55, 11029 (1997). 
[57] M. Rigol, V. Dunjko, and M. Olshanii, Thermalization and Its Mechanism for Generic Isolated Quantum Systems, Nature (London) 452, 854 (2008).

[58] T. Barthel and U. Schollwöck, Dephasing and the Steady State in Quantum Many-Particle Systems, Phys. Rev. Lett. 100, 100601 (2008).

[59] B. Feigin, E. Frenkel, and N. Reshetikhin, Gaudin Model, Bethe Ansatz and Critical Level, Commun. Math. Phys. 166, 27 (1994).

[60] E. Iyoda, H. Katsura, and T. Sagawa, Effective Dimension, Level Statistics, and Integrability of Sachdev-Ye-Kitaev-like Models, Phys. Rev. D 98, 086020 (2018).

[61] We initialize each spin $i$ in a randomly chosen (with equal probability $p=1 / 6$ ) eigenstate of either $\hat{S}_{i}^{x}, \hat{S}_{i}^{y}$, or $\hat{S}_{i}^{z}$.

[62] R. Nandkishore and D. A. Huse, Many-Body Localization and Thermalization in Quantum Statistical Mechanics, Annu. Rev. Condens. Matter Phys. 6, 15 (2015).

[63] J. Schliemann, Spins Coupled to a Spin Bath: From Integrability to Chaos, Phys. Rev. B 81, 081301(R) (2010).

[64] H. K. Owusu, K. Wagh, and E. A. Yuzbashyan, The Link between Integrability, Level Crossings and Exact Solution in Quantum Models, J. Phys. A 42, 035206 (2009).

[65] J. A. Scaramazza, B. S. Shastry, and E. A. Yuzbashyan, Integrable Matrix Theory: Level Statistics, Phys. Rev. E 94, 032106 (2016).

[66] G. Benettin, L. Galgani, A. Giorgilli, and J.-M. Strelcyn, Lyapunov Characteristic Exponents for Smooth Dynamical Systems and for Hamiltonian Systems; a Method for Computing All of Them. Part 2: Numerical Application, Meccanica 15, 21 (1980).

[67] I. Goldhirsch, P.-L. Sulem, and S. A. Orszag, Stability and Lyapunov Stability of Dynamical Systems: A Differential Approach and a Numerical Method, Physica D (Amsterdam) 27, 311 (1987).

[68] The main difference between the classical and quantum SVD results is that the classical singular values are considerably larger than the quantum ones (compare Figs. 2 and 7). This is because the expectation value of $O_{a}$ is evaluated on a single classical configuration in the former case, while the quantum expectation value is a result of a coherent average.

[69] We have checked that this persists up to $T=10^{6} \mathrm{~J}^{-2}$. Nonetheless, we find that these plateaus eventually decay for a finite-size system, albeit after a very long time. We leave the quantitative analysis for future work.

[70] A. I. Larkin and Y. N. Ovchinnikov, Quasiclassical Method in the Theory of Superconductivity, J. Exp. Theor. Phys. 28, 1200 (1969).

[71] S. H. Shenker and D. Stanford, Black Holes and the Butterfly Effect, J. High Energy Phys. 3 (2014) 067.

[72] P. Hosur, X.-L. Qi, D. A. Roberts, and B. Yoshida, Chaos in Quantum Channels, J. High Energy Phys. 2 (2016) 004.

[73] M. Gärttner, J. G. Bohnet, A. Safavi-Naini, M. L. Wall, J. J. Bollinger, and A. M. Rey, Measuring Out-of-Time-Order Correlations and Multiple Quantum Spectra in a TrappedIon Quantum Magnet, Nat. Phys. 13, 781 (2017).

[74] B. Vermersch, A. Elben, L. M. Sieberer, N. Y. Yao, and P. Zoller, Probing Scrambling Using Statistical Correlations between Randomized Measurements, Phys. Rev. X 9, 021061 (2019).

[75] R. J. Lewis-Swan, A. Safavi-Naini, J. J. Bollinger, and A. M. Rey, Unifying Scrambling, Thermalization and Entanglement through Measurement of Fidelity Out-of-TimeOrder Correlators in the Dicke Model, Nat. Commun. 10, 1581 (2019).

[76] J. S. Cotler, D. Ding, and G. R. Penington, Out-of-TimeOrder Operators and the Butterfly Effect, Ann. Phys. (Amsterdam) 396, 318 (2018).

[77] B. Swingle and N. Y. Halpern, Resilience of Scrambling Measurements, Phys. Rev. A 97, 062113 (2018).

[78] J. K. Eastman, J. J. Hope, and A. R. R. Carvalho, Tuning Quantum Measurements to Control Chaos, Sci. Rep. 7, 44684 (2017).

[79] Z. Xu, L. Pedro García-Pintos, A. Chenu, and A. del Campo, Extreme Decoherence and Quantum Chaos, Phys. Rev. Lett. 122, 014103 (2019).

[80] S. Habib, K. Shizume, and W. H. Zurek, Decoherence, Chaos, and the Correspondence Principle, Phys. Rev. Lett. 80, 4361 (1998).

[81] M. V. Berry and M. Tabor, Level Clustering in the Regular Spectrum, Proc. R. Soc. A 356, 375 (1977).

[82] O. Bohigas, M. J. Giannoni, and C. Schmit, Characterization of Chaotic Quantum Spectra and Universality of Level Fluctuation Laws, Phys. Rev. Lett. 52, 1 (1984).

[83] A. G. Bytsko, On Integrable Hamiltonians for Higher Spin XXZ Chain, J. Math. Phys. (N.Y.) 44, 3698 (2003). 